\title{
GEOMETRY OF REGULAR MODULES OVER CANONICAL ALGEBRAS
}

\author{
GRZEGORZ BOBIŃSKI
}

Dedicated to the memory of Professor Stanistaw Balcerzyk

\begin{abstract}
We classify canonical algebras such that for every dimension vector of a regular module the corresponding module variety is normal (respectively, a complete intersection). We also prove that for the dimension vectors of regular modules normality is equivalent to irreducibility.
\end{abstract}

\section{INTRODUCTION AND MAIN RESULT}

Throughout the paper $k$ is a fixed algebraically closed field. By an algebra we always mean a finite dimensional algebra over $k$ and by a module a finite dimensional left module.

In $[20,3.7]$ Ringel introduced a class of so-called canonical algebras (see Subsection 2.4 for a definition). A canonical algebra $\Lambda$ depends on a sequence $\left(m_{1}, \ldots, m_{n}\right)$, $n>2$, of positive integers greater than 1 , and on a sequence $\left(\lambda_{3}, \ldots, \lambda_{n}\right)$ of pairwise distinct nonzero elements of $k$. In the above situation we say that $\Lambda$ is a canonical algebra of type $\left(m_{1}, \ldots, m_{n}\right)$. These algebras play a prominent role in the representation theory of algebras. For example their module categories serve as model categories for module categories of algebras admitting separating tubular families (see $[17,21]$ ). The module categories of canonical algebras are derived equivalent to the categories of coherent sheaves over weighted projective lines (see [11]). Moreover, according to [13, Theorem 3.1] every quasi-titled algebra is derived equivalent either to a hereditary algebra or to a canonical one.

An important and interesting direction of research in the representation theory of algebras is the study of varieties $\bmod _{\Lambda}(\mathbf{d})$ of $\Lambda$-modules of dimension vector $\mathbf{d}$ (see Section 3.1), where $\mathbf{d}$ is an element of the Grothendieck group $K_{0}(\Lambda)$ (for some reviews of results see for example $[7,12,16]$ ). In particular, varieties of modules over canonical algebras have been studied. In [2] Skowroński and the author proved that if $\Lambda$ is a tame canonical algebra and $\mathbf{d}$ is the dimension vector of an indecomposable $\Lambda$-module, then $\bmod _{\Lambda}(\mathbf{d})$ is a complete intersection with at most 2 irreducible components. It was also shown that in the above case irreducibility of $\bmod _{\Lambda}(\mathbf{d})$ is equivalent to normality.

For a canonical algebra $\Lambda$ one may distinguish so-called regular modules (see Subsection 2.5). This class of modules also received special attention from a geometric

Received by the editors May 16, 2005 and, in revised form, October 4, 2005.

2000 Mathematics Subject Classification. Primary 16G20; Secondary 14L30.

Key words and phrases. Canonical algebra, module variety, normal variety, complete intersection. 
point of view. Skowroński and the author showed in [3] that if $\mathbf{d}$ is the dimension vector of a regular module over a tame canonical algebra $\Lambda$, then the corresponding variety is an irreducible and normal complete intersection. Similar results for special cases of wild canonical algebras were obtained by Barot and Schröer in [1]. It is also worth mentioning that if $\mathbf{d}$ is the dimension vector of a regular module over a canonical algebra, then descriptions of the semi-invariants with respect to the natural action of GL(d) were given independently by Skowroński and Weyman in $[22]$ and Domokos and Lenzing in $[9,10]$.

Our first theorem generalizes to regular modules over arbitrary canonical algebra a result obtained for indecomposable modules over tame canonical algebra in [2].

Theorem 1.1. Let $\Lambda$ be a canonical algebra and let $\mathbf{d}$ be the dimension vector of a regular $\Lambda$-module. Then $\bmod _{\Lambda}(\mathbf{d})$ is normal if and only if it is irreducible.

Let $a(\mathbf{d})=\operatorname{dim} \mathrm{GL}(\mathbf{d})-\langle\mathbf{d}, \mathbf{d}\rangle$ for $\mathbf{d} \in K_{0}(\Lambda)$, where $\mathrm{GL}(\mathbf{d})$ is the corresponding product of general linear groups (see Subsection 3.1) and $\langle-,-\rangle: K_{0}(\Lambda) \times K_{0}(\Lambda) \rightarrow$ $\mathbb{Z}$ is the Ringel bilinear form (see Subsection 2.3). We have the following criterion for a complete intersection.

Theorem 1.2. Let $\Lambda$ be a canonical algebra and let $\mathbf{d}$ be the dimension vector of a regular $\Lambda$-module. Then $\bmod _{\Lambda}(\mathbf{d})$ is a complete intersection if and only if $\operatorname{dim} \bmod _{\Lambda}(\mathbf{d})=a(\mathbf{d})$.

In Propositions 4.3 and 4.5 we show how the above theorems can be translated into numeric properties of the Ringel form.

Our aim in this paper is to classify canonical algebras such that the corresponding module varieties have "good" geometric properties for all dimension vectors of regular modules. It is done in the following theorem.

Theorem 1.3. Let $\Lambda$ be a canonical algebra of type $\left(m_{1}, \ldots, m_{n}\right)$.

(1) The varieties $\bmod _{\Lambda}(\mathbf{d})$ are complete intersections for all dimension vectors $\mathbf{d}$ of regular $\Lambda$-modules if and only if

$$
\frac{1}{m_{1}-1}+\cdots+\frac{1}{m_{n}-1} \geq 2 n-5 .
$$

(2) The varieties $\bmod _{\Lambda}(\mathbf{d})$ are normal for all dimension vectors $\mathbf{d}$ of regular $\Lambda$-modules if and only if

$$
\frac{1}{m_{1}-1}+\cdots+\frac{1}{m_{n}-1}>2 n-5 .
$$

Recall that if $\Lambda$ is a canonical algebra of type $\left(m_{1}, \ldots, m_{n}\right)$, then $\Lambda$ is of tame (respectively, domestic) representation type if and only if

$$
\frac{1}{m_{1}}+\cdots+\frac{1}{m_{n}} \geq n-2(>n-2) .
$$

A natural assumption when dealing with geometric problems is that $\mathbf{d}$ is the dimension vector of a sincere module $M$ (i.e., every simple module occurs as a composition factor of $M$ ). Such dimension vectors are also called sincere. We have the corresponding result in this case.

Theorem 1.4. Let $\Lambda$ be a canonical algebra of type $\left(m_{1}, \ldots, m_{n}\right)$.

(1) The varieties $\bmod _{\Lambda}(\mathbf{d})$ are complete intersections for all dimension vectors $\mathbf{d}$ of sincere regular $\Lambda$-modules if and only if

$$
\frac{1}{m_{1}-1}+\cdots+\frac{1}{m_{n}-1} \geq 2 n-5 .
$$


(2) The varieties $\bmod _{\Lambda}(\mathbf{d})$ are normal for all dimension vectors $\mathbf{d}$ of sincere regular $\Lambda$-modules if and only if either

$$
\begin{array}{r}
\frac{1}{m_{1}-1}+\cdots+\frac{1}{m_{n}-1}>2 n-5, \\
\text { or } n=5 \text { and } m_{i}=2 \text { for all } i=1, \ldots, 5 \text {. }
\end{array}
$$

Let $\mathbf{h}$ be the dimension vector of the multiplicity free sincere semi-simple $\Lambda$ module (see Subsection 2.4). It can be observed from $[3,10,22]$ that results about $\bmod _{\Lambda}(\mathbf{d})$ depend on whether there exists a regular $\Lambda$-module $M$ of dimension vector $\mathbf{d}$ which has a direct summand of dimension vector $\mathbf{h}$. Let $\mathbf{R}^{\prime}$ be the set of all such dimension vectors (see also Subsection 2.6).

Theorem 1.5. Let $\Lambda$ be a canonical algebra of type $\left(m_{1}, \ldots, m_{n}\right)$.

(1) The varieties $\bmod _{\Lambda}(\mathbf{d})$ are complete intersections for all dimension vectors $\mathbf{d} \in \mathbf{R}^{\prime}$ if and only if

$$
\frac{1}{m_{1}-1}+\cdots+\frac{1}{m_{n}-1} \geq 2 n-5 .
$$

(2) The varieties $\bmod _{\Lambda}(\mathbf{d})$ are normal for all dimension vectors $\mathbf{d} \in \mathbf{R}^{\prime}$ if and only if

$$
\frac{1}{m_{1}-1}+\cdots+\frac{1}{m_{n}-1} \geq 2 n-5 .
$$

We exclude from our considerations the case of canonical algebras of type $\left(m_{1}, m_{2}\right)$, since in this case the module varieties are just affine spaces. However, the above theorems are also trivially satisfied in this case, if we set $\frac{1}{0}=\infty$.

The paper is organized as follows. In Section 2 we present necessary facts about canonical algebras. In Section 3 we collect some useful facts about varieties of modules, while in Section 4 we prove Theorems 1.1 and 1.2, and show how to reduce the proofs of Theorems 1.3, 1.4 and 1.5 to questions about properties of the Ringel form. Next in Section 5 we prove inequalities which show that for canonical algebras satisfying the conditions of Theorems 1.3, 1.4 and 1.5, the corresponding module varieties have the required properties. On the other hand, in Section 6 we present examples showing that the above statements do not hold for the remaining canonical algebras.

The results presented in this paper were obtained while the author held a one year post-doc position at the University of Bern. The author gratefully acknowledges the support from the Schweizerischer Nationalfonds and the Polish Scientific Grant KBN No. 1 P03A 018 27. The author also expresses his gratitude to Professor Riedtmann for discussions, which were an inspiration for this research.

\section{FACts ABOUt CANONICAL ALGEBras}

Throughout the paper, by $\mathbb{N}$ and $\mathbb{Z}$ we denote the sets of nonnegative integers and integers, respectively. If $i, j \in \mathbb{Z}$, then $[i, j]$ denotes the set of all $l \in \mathbb{Z}$ such that $i \leq l \leq j$.

2.1. Recall that by a quiver $\Delta$ we mean a finite set $\Delta_{0}$ of vertices and a finite set $\Delta_{1}$ of arrows together with two maps $s, t: \Delta_{1} \rightarrow \Delta_{0}$, which assign to an arrow $\gamma \in \Delta_{1}$ its starting and terminating vertex, respectively. By a path of length $m \geq 1$ in $\Delta$ we mean a sequence $\sigma=\gamma_{1} \cdots \gamma_{m}$ of arrows such that $s \gamma_{i}=t \gamma_{i+1}$ for $i \in[1, m-1]$. We write $s \sigma$ and $t \sigma$ for $s \gamma_{m}$ and $t \gamma_{1}$, respectively. For each vertex $x$ of $\Delta$ we introduce a path $x$ of length 0 such that $s x=x=t x$. We only consider quivers without 
oriented cycles, i.e., we assume that there exists no path $\sigma$ of positive length such that $t \sigma=s \sigma$.

With a quiver $\Delta$ we associate its path algebra $k \Delta$, which as a $k$-vector space has a basis formed by all paths in $\Delta$ and whose multiplication is induced by the composition of paths. By a relation $\rho$ in $\Delta$ we mean a linear combination of paths of length at least 2 with the same starting and terminating vertex. This common starting vertex is denoted by $s \rho$ and the common terminating vertex by $t \rho$. A set $R$ of relations is called minimal if for every $\rho \in R, \rho$ does not belong to the ideal $\langle R \backslash\{\rho\}\rangle$ of $k \Delta$ generated by $R \backslash\{\rho\}$. A pair $(\Delta, R)$ consisting of a quiver $\Delta$ and a minimal set of relations $R$ is called a bound quiver. If $(\Delta, R)$ is a bound quiver, then the algebra $k \Delta /\langle R\rangle$ is called the path algebra of $(\Delta, R)$.

2.2. Let $\Lambda$ be the path algebra of a bound quiver $(\Delta, R)$. It is known that the category $\bmod _{\Lambda}$ of $\Lambda$-modules is equivalent to the category of representations of $(\Delta, R)$ (see for example $[20,2.1]$ ). Recall, that by a representation of $(\Delta, R)$ we mean a collection $\left(M_{x}, M_{\gamma}\right)_{x \in \Delta_{0}, \gamma \in \Delta_{1}}$ of finite dimensional $k$-vector spaces $M_{x}$, $x \in \Delta_{0}$, and $k$-linear maps $M_{\gamma}: M_{s \gamma} \rightarrow M_{t \gamma}, \gamma \in \Delta_{1}$, such that $M_{\rho}=0$ for all $\rho \in R$. Here, if $\sigma=\gamma_{1} \cdots \gamma_{m}$ is a path in $\Delta$, then we write $M_{\sigma}=M_{\gamma_{1}} \cdots M_{\gamma_{m}}$, and if $\rho=\lambda_{1} \sigma_{1}+\cdots+\lambda_{n} \sigma_{n}$ is a relation in $\Delta$, then $M_{\rho}=\lambda_{1} M_{\sigma_{1}}+\cdots+\lambda_{n} M_{\sigma_{n}}$. If $M$ and $N$ are two representations of $(\Delta, R)$, then by a morphism $f: M \rightarrow N$ we mean a collection $\left(f_{x}\right)_{x \in \Delta_{0}}$ of linear maps $f_{x}: M_{x} \rightarrow N_{x}, x \in \Delta_{0}$, such that $f_{t \gamma} M_{\gamma}=N_{\gamma} f_{s \gamma}$ for all $\gamma \in \Delta_{1}$. From now on we identify $\Lambda$-modules with representations of $(\Delta, R)$. In particular, for each $\Lambda$-module $M$ we define its dimension vector $\operatorname{dim} M \in \mathbb{N}^{\Delta_{0}}$ by $(\operatorname{dim} M)_{x}=\operatorname{dim}_{k} M_{x}, x \in \Delta_{0}$.

2.3. Let $\Lambda$ be the path algebra of a bound quiver $(\Delta, R)$. For a vertex $x$ of $\Delta_{0}$ we denote by $\mathbf{e}_{x}$ the element of the canonical basis of $\mathbb{Z}^{\Delta_{0}}$ corresponding to $x$. For $\mathbf{d} \in \mathbb{Z}^{\Delta_{0}}$ we write $\mathbf{d}=\sum_{x \in \Delta_{0}} d_{x} \mathbf{e}_{x}$. Assume that gl. $\operatorname{dim} \Lambda \leq 2$. We have the Ringel bilinear form $\langle-,-\rangle: \mathbb{Z}^{\Delta_{0}} \times \mathbb{Z}^{\Delta_{0}} \rightarrow \mathbb{Z}$ defined by

$$
\left\langle\mathbf{d}^{\prime}, \mathbf{d}^{\prime \prime}\right\rangle=\sum_{x \in \Delta_{0}} d_{x}^{\prime} d_{x}^{\prime \prime}-\sum_{\gamma \in \Delta_{1}} d_{s \gamma}^{\prime} d_{t \gamma}^{\prime \prime}+\sum_{\rho \in R} d_{s \rho}^{\prime} d_{t \rho}^{\prime \prime} .
$$

It is known (see $[4,2.2]$ ), that if $M$ and $N$ are $\Lambda$-modules, then

$$
\langle\operatorname{dim} M, \operatorname{dim} N\rangle=[M, N]-[M, N]^{1}+[M, N]^{2},
$$

where following Bongartz [5] we write $[M, N]=\operatorname{dim}_{k} \operatorname{Hom}_{\Lambda}(M, N),[M, N]^{1}=$ $\operatorname{dim}_{k} \operatorname{Ext}_{\Lambda}^{1}(M, N)$ and $[M, N]^{2}=\operatorname{dim}_{k} \operatorname{Ext}_{\Lambda}^{2}(M, N)$.

2.4. Let $\mathbf{m}=\left(m_{1}, \ldots, m_{n}\right), n \geq 3$, be a sequence of integers greater than 1 and let $\boldsymbol{\lambda}=\left(\lambda_{3}, \ldots, \lambda_{n}\right)$ be a sequence of pairwise distinct nonzero elements of $k$. We define $\Lambda(\mathbf{m}, \boldsymbol{\lambda})$ as the path algebra of the quiver $\Delta(\mathbf{m})$

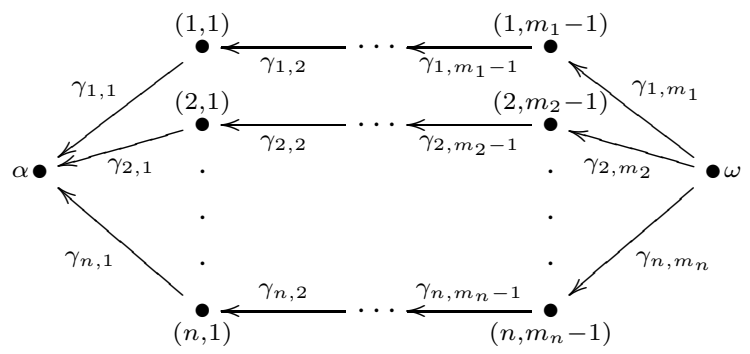


bound by relations

$$
\gamma_{1,1} \cdots \gamma_{1, m_{1}}+\lambda_{i} \gamma_{2,1} \cdots \gamma_{2, m_{2}}-\gamma_{i, 1} \cdots \gamma_{i, m_{i}}, i \in[3, n] .
$$

The algebras of the above form are called canonical. In particular, we call $\Lambda(\mathbf{m}, \boldsymbol{\lambda})$ a canonical algebra of type $\mathbf{m}$. It is well known (see for example [14, III.4]) that gl. $\operatorname{dim} \Lambda(\mathbf{m}, \boldsymbol{\lambda})=2$. If $\mathbf{m}$ and $\boldsymbol{\lambda}$ are fixed, then we usually write $\Lambda$ and $\Delta$ instead of $\Lambda(\mathbf{m}, \boldsymbol{\lambda})$ and $\Delta(\mathbf{m})$, respectively. From now until the end of the section we assume that $\Lambda=\Lambda(\mathbf{m}, \boldsymbol{\lambda})$ is a fixed canonical algebra.

We write $\mathbf{e}_{i, j}$ instead of $\mathbf{e}_{(i, j)}$ for $i \in[1, n]$ and $j \in\left[1, m_{i}-1\right]$. For future convenience for $i \in[1, n]$ by $(i, 0)$ and $\left(i, m_{i}\right)$ we mean $\alpha$ and $\omega$, respectively. Moreover, if $i \in[1, n]$ and $j \in\left[0, m_{i}\right]$, then we write $d_{i, j}$ instead of $d_{(i, j)}$. Let $\mathbf{h}=\sum_{x \in \Delta_{0}} \mathbf{e}_{x}$. For $i \in[1, n]$, we put $\mathbf{e}_{i, 0}=\mathbf{e}_{i, m_{i}}=\mathbf{h}-\sum_{j \in\left[1, m_{i}-1\right]} \mathbf{e}_{i, j}$. Note that

$$
\left\langle\mathbf{e}_{i, j}, \mathbf{d}\right\rangle=d_{i, j}-d_{i, j-1}, i \in[1, n], j \in\left[1, m_{i}\right],
$$

and

$$
\left\langle\mathbf{d}, \mathbf{e}_{i, j}\right\rangle=d_{i, j}-d_{i, j+1}, i \in[1, n], j \in\left[0, m_{i}-1\right],
$$

and consequently $\langle\mathbf{h}, \mathbf{d}\rangle=d_{\omega}-d_{\alpha}=-\langle\mathbf{d}, \mathbf{h}\rangle$, for all $\mathbf{d} \in \mathbb{Z}^{\Delta_{0}}$.

2.5. Let $\mathcal{P}(\mathcal{R}, \mathcal{Q}$, respectively) be the subcategory of all $\Lambda$-modules which are direct sums of indecomposable $\Lambda$-modules $X$ such that

$$
\langle\operatorname{dim} X, \mathbf{h}\rangle>0 \quad(\langle\operatorname{dim} X, \mathbf{h}\rangle=0,\langle\operatorname{dim} X, \mathbf{h}\rangle<0, \text { respectively }) .
$$

The $\Lambda$-modules belonging to $\mathcal{R}$ are called regular. We have the following properties of the above decomposition of $\bmod _{\Lambda}($ see $[20,3.7])$.

First, $[N, M]=0$ and $[M, N]^{1}=0$, if either $N \in \mathcal{R} \vee \mathcal{Q}$ and $M \in \mathcal{P}$, or $N \in \mathcal{Q}$ and $M \in \mathcal{P} \vee \mathcal{R}$. Here, for two subcategories $\mathcal{X}$ and $\mathcal{Y}$ of $\bmod _{\Lambda}$, we denote by $\mathcal{X} \vee \mathcal{Y}$ the additive closure of their union. Second, $\mathcal{R}$ decomposes into a $\mathbb{P}^{1}(k)$ family $\coprod_{\lambda \in \mathbb{P}^{1}(k)} \mathcal{R}_{\lambda}$ of uniserial categories. If $\lambda \in \mathbb{P}^{1}(k) \backslash\left\{\lambda_{1}, \ldots, \lambda_{n}\right\}$, where $\lambda_{1}=0$ and $\lambda_{2}=\infty$, then there is a unique simple object in $\mathcal{R}_{\lambda}$, and its dimension vector is $\mathbf{h}$. On the other hand, if $\lambda=\lambda_{i}$ for $i \in[1, n]$, then there are $m_{i}$ simple objects in $\mathcal{R}_{\lambda_{i}}$ and their dimension vectors are $\mathbf{e}_{i, j}, j \in\left[1, m_{i}\right]$. Finally, one knows that $\operatorname{pd}_{\Lambda} M \leq 1$ for $M \in \mathcal{P} \vee \mathcal{R}$ and $\operatorname{id}_{\Lambda} N \leq 1$ for $N \in \mathcal{R} \vee \mathcal{Q}$.

2.6. We denote by $\mathbf{P}, \mathbf{R}$ and $\mathbf{Q}$ the sets of the dimension vectors of the $\Lambda$-modules belonging to $\mathcal{P}, \mathcal{R}$ and $\mathcal{Q}$, respectively. Note that $\mathbf{d} \in \mathbf{R}$ if and only if

$$
\mathbf{d}=p \mathbf{h}+\sum_{i \in[1, n]} \sum_{j \in\left[1, m_{i}\right]} p_{i, j} \mathbf{e}_{i, j}
$$

for some nonnegative integers $p$ and $p_{i, j}, i \in[1, n], j \in\left[1, m_{i}\right]$. We know from [20, 3.7], that if $\mathbf{d} \in \mathbf{P}, \mathbf{d} \neq 0$, then $d_{\alpha}>d_{\omega} \geq 0$ and $d_{i, j} \geq d_{i, j+1}$ for all $i \in[1, n]$ and $j \in\left[0, m_{i}-1\right]$. We now show the converse. Let $\mathbf{d}$ be as above. Fix $\lambda_{0} \in$ $\mathbb{P}^{1}(k) \backslash\left\{\lambda_{1}, \ldots, \lambda_{n}\right\}$. It is easy to see that there exists $M \in \mathcal{P} \vee \mathcal{R}_{\lambda_{0}}$ of dimension vector $\mathbf{d}$. Indeed, it is enough to write $\mathbf{d}=\mathbf{d}^{\prime}+\mathbf{d}^{\prime \prime}$, where $\mathbf{d}^{\prime \prime}=d_{\omega} \mathbf{h}$. Then obviously there is $M^{\prime \prime} \in \mathcal{R}_{\lambda_{0}}$ of dimension vector $\mathbf{d}^{\prime \prime}$, and one can easily construct $M^{\prime} \in \mathcal{P}$ of dimension vector $\mathbf{d}^{\prime}$, since $d_{\omega}^{\prime}=0$. Since $\left[N^{\prime}, N^{\prime \prime}\right]=0$ for $N^{\prime} \in \coprod_{\lambda \neq \lambda_{0}} \mathcal{R}_{\lambda} \vee \mathcal{Q}$ and $N^{\prime \prime} \in \mathcal{P} \vee \mathcal{R}_{\lambda_{0}}$, it follows that $\mathcal{P} \vee \mathcal{R}_{\lambda_{0}}$ is extension closed. In particular, if we assume that the dimension of the endomorphism ring of $M$ is minimal possible, then $M=M^{\prime} \oplus M^{\prime \prime}$ for $M^{\prime} \in \mathcal{P}$ and $M^{\prime \prime} \in \mathcal{R}_{\lambda_{0}}$ such that $\left[M^{\prime \prime}, M^{\prime}\right]^{1}=0$ (see for example $[20,2.3])$. On the other hand, $M^{\prime \prime}=p \mathbf{h}$ for a nonnegative integer $p$, and 
$\left[M^{\prime \prime}, M^{\prime}\right]^{1}=-\langle p \mathbf{h}, \mathbf{d}-p \mathbf{h}\rangle=p\left(d_{\alpha}-d_{\omega}\right)>0$, if $p>0$. Thus $p=0, M^{\prime \prime}=0$, and $M=M^{\prime} \in \mathcal{P}$.

Dually, $\mathbf{d} \in \mathbf{Q}, \mathbf{d} \neq 0$, if and only if $0 \leq d_{\alpha}<d_{\omega}$ and $d_{i, j-1} \leq d_{i, j}$ for all $i \in[1, n]$ and $j \in\left[1, m_{i}\right]$. Thus, each $\mathbf{d} \in \mathbf{Q}$ can be written in a form

$$
\mathbf{d}=p \mathbf{h}+\sum_{i \in[1, n]} \sum_{j \in\left[1, m_{i}-1\right]} p_{i, j} \mathbf{e}_{i, j}+p_{\omega} \mathbf{e}_{\omega}
$$

for some nonnegative integers $p, p_{\omega}$ and $p_{i, j}, i \in[1, n], j \in\left[1, m_{i}-1\right]$. Consequently, $\mathbf{d} \in \mathbf{R}+\mathbf{Q}$ if and only if

$$
\mathbf{d}=p \mathbf{h}+\sum_{i \in[1, n]} \sum_{j \in\left[1, m_{i}\right]} p_{i, j} \mathbf{e}_{i, j}+p_{\omega} \mathbf{e}_{\omega}
$$

for some nonnegative integers $p, p_{\omega}$ and $p_{i, j}, i \in[1, n], j \in\left[1, m_{i}\right]$. In particular, if $\mathbf{d} \in \mathbf{R}+\mathbf{Q}$, then there exists a unique presentation

$$
\mathbf{d}=p^{\mathbf{d}} \mathbf{h}+\sum_{i \in[1, n]} \sum_{j \in\left[1, m_{i}\right]} p_{i, j}^{\mathbf{d}} \mathbf{e}_{i, j}+p_{\omega}^{\mathbf{d}} \mathbf{e}_{\omega}
$$

such that $p^{\mathbf{d}}, p_{\omega}^{\mathbf{d}}$ and $p_{i, j}^{\mathbf{d}}, i \in[1, n], j \in\left[1, m_{i}\right]$, are nonnegative integers, and for each $i \in[1, n]$ there exists $j \in\left[1, m_{i}\right]$ such that $p_{i, j}^{\mathbf{d}}=0$. Note that $\mathbf{d} \in \mathbf{R}$ if and only if $p_{\omega}^{\mathbf{d}}=0$. Moreover, $\mathbf{d} \in \mathbf{R}^{\prime}$ if and only if $p_{\omega}^{\mathbf{d}}=0$ and $p^{\mathbf{d}} \neq 0$. Recall that by $\mathbf{R}^{\prime}$ we denote the set of all dimension vectors of regular $\Lambda$-modules which have a direct summand of dimension vector $\mathbf{h}$.

\section{VARIETIES OF MODULES}

Throughout this section $\Lambda$ is the path algebra of a bound quiver $(\Delta, R)$ of global dimension at most 2 .

3.1. For $\mathbf{d}^{\prime}, \mathbf{d}^{\prime \prime} \in \mathbb{N}^{\Delta_{0}}$, let $\mathbb{A}\left(\mathbf{d}^{\prime}, \mathbf{d}^{\prime \prime}\right)=\prod_{\gamma \in \Delta_{1}} \mathbb{M}\left(d_{t \gamma}^{\prime}, d_{s \gamma}^{\prime \prime}\right)$, where by $\mathbb{M}(p, q)$ we denote the space of $p \times q$-matrices with coefficients in $k$. For a dimension vector $\mathbf{d} \in \mathbb{N}^{\Delta_{0}}, M \in \mathbb{A}(\mathbf{d}, \mathbf{d})$ and a path $\sigma=\gamma_{1} \cdots \gamma_{m}$ of positive length, we put $M_{\sigma}=$ $M_{\gamma_{1}} \cdots M_{\gamma_{m}}$. We extend this notation to relations in the standard way. We denote by $\bmod _{\Lambda}(\mathbf{d})$ the set of all $M \in \mathbb{A}(\mathbf{d}, \mathbf{d})$, such that $M_{\rho}=0$ for all $\rho \in R$. Obviously, $\bmod _{\Lambda}(\mathbf{d})$ is an affine variety. Note that every point $M$ of $\bmod _{\Lambda}(\mathbf{d})$ determines a $\Lambda$-module of dimension vector $\mathbf{d}$ (by taking $M_{x}=k^{d_{x}}$ for $x \in \Delta_{0}$ ), which we also denote by $M$, and every $\Lambda$-module of dimension vector $\mathbf{d}$ is isomorphic to $M$ for some $M \in \bmod _{\Lambda}(\mathbf{d})$. We call $\bmod _{\Lambda}(\mathbf{d})$ the variety of $\Lambda$-modules of dimension vector $\mathbf{d}$. Note that $a(\mathbf{d})$ defined in Section 1 can be calculated as follows:

$$
a(\mathbf{d})=\sum_{\gamma \in \Delta_{1}} d_{s \gamma} d_{t \gamma}-\sum_{\rho \in R} d_{s \rho} d_{t \rho},
$$

hence $a(\mathbf{d})$ is just the dimension of $\mathbb{A}(\mathbf{d}, \mathbf{d})$ minus the number of equations defining $\bmod _{\Lambda}(\mathbf{d})$. In particular, the dimension of each irreducible component of $\bmod _{\Lambda}(\mathbf{d})$ is at least $a(\mathbf{d})$.

The product $\mathrm{GL}(\mathbf{d})=\prod_{x \in \Delta_{0}} \mathrm{GL}\left(d_{x}\right)$ of general linear groups acts on $\bmod _{\Lambda}(\mathbf{d})$ by conjugations

$$
(g \cdot M)_{\gamma}=g_{t \gamma} M_{\gamma} g_{s \gamma}^{-1}, \gamma \in \Delta_{1},
$$

for $g \in \mathrm{GL}(\mathbf{d})$ and $M \in \bmod _{\Lambda}(\mathbf{d})$. The orbits with respect to this action correspond bijectively to the isomorphism classes of $\Lambda$-modules of dimension vector $\mathbf{d}$. 
3.2. We now present a construction investigated in $[5,2.1]$ by Bongartz. Fix $\mathbf{d}^{\prime}, \mathbf{d}^{\prime \prime} \in$ $\mathbb{N}^{\Delta_{0}}, M^{\prime} \in \bmod _{\Lambda}\left(\mathbf{d}^{\prime}\right)$ and $M^{\prime \prime} \in \bmod _{\Lambda}\left(\mathbf{d}^{\prime \prime}\right)$. For $Z \in \mathbb{A}\left(\mathbf{d}^{\prime}, \mathbf{d}^{\prime \prime}\right)$ and a path $\sigma=$ $\gamma_{1} \cdots \gamma_{m}$ of positive length, let

$$
Z_{\sigma}=\sum_{i \in[1, m]} M_{\gamma_{1}}^{\prime} \cdots M_{\gamma_{i-1}}^{\prime} Z_{\gamma_{i}} M_{\gamma_{i+1}}^{\prime \prime} \cdots M_{\gamma_{m}}^{\prime \prime}
$$

If $\rho=\sum_{i \in[1, n]} \lambda_{i} \sigma_{i}$ is a relation in $\Delta$, then $Z_{\rho}=\sum_{i \in[1, n]} \lambda_{i} Z_{\sigma_{i}}$. We define $Z\left(M^{\prime \prime}, M^{\prime}\right)$ as the set of all $Z \in \mathbb{A}\left(\mathbf{d}^{\prime}, \mathbf{d}^{\prime \prime}\right)$ such that $Z_{\rho}=0$ for all $\rho \in R$. For $Z \in Z\left(M^{\prime \prime}, M^{\prime}\right)$, let $M \in \mathbb{A}\left(\mathbf{d}^{\prime}+\mathbf{d}^{\prime \prime}, \mathbf{d}^{\prime}+\mathbf{d}^{\prime \prime}\right)$ be given by

$$
M_{\gamma}=\left[\begin{array}{cc}
M_{\gamma}^{\prime} & Z_{\gamma} \\
0 & M_{\gamma}^{\prime \prime}
\end{array}\right], \gamma \in \Delta_{1}
$$

Then $M \in \bmod _{\Lambda}\left(\mathbf{d}^{\prime}+\mathbf{d}^{\prime \prime}\right)$, and we have a short exact sequence

$$
0 \rightarrow M^{\prime} \stackrel{f}{\rightarrow} M \stackrel{g}{\rightarrow} M^{\prime \prime} \rightarrow 0,
$$

with the maps $f$ and $g$ given by the canonical injections $k^{d_{x}^{\prime}} \rightarrow k^{d_{x}^{\prime}+d_{x}^{\prime \prime}}, x \in \Delta_{0}$, and the canonical surjections $k^{d_{x}^{\prime}+d_{x}^{\prime \prime}} \rightarrow k^{d_{x}^{\prime \prime}}, x \in \Delta_{0}$, respectively. On the other hand, for every short exact sequence $\varepsilon$ of the form

$$
0 \rightarrow M^{\prime} \rightarrow M \rightarrow M^{\prime \prime} \rightarrow 0,
$$

there exists a (nonunique) element of $Z\left(M^{\prime \prime}, M^{\prime}\right)$ such that the corresponding short exact sequence is isomorphic to $\varepsilon$. More precisely, the map $Z\left(M^{\prime \prime}, M^{\prime}\right) \rightarrow$ $\operatorname{Ext}_{\Lambda}^{1}\left(M^{\prime \prime}, M^{\prime}\right)$ described above is a surjective linear map. The kernel of this map consists of $Z \in Z\left(M^{\prime \prime}, M^{\prime}\right)$ such that the corresponding sequence splits, i.e., there exists $h \in \mathbb{V}\left(\mathbf{d}^{\prime}, \mathbf{d}^{\prime \prime}\right)=\prod_{x \in \Delta_{0}} \mathbb{M}\left(d_{x}^{\prime}, d_{x}^{\prime \prime}\right)$ such that $Z_{\gamma}=M_{\gamma}^{\prime} h_{s \gamma}-h_{t \gamma} M_{\gamma}^{\prime \prime}$ for all $\gamma \in \Delta_{1}$. Consequently,

$$
\operatorname{dim}_{k} Z\left(M^{\prime \prime}, M^{\prime}\right)=\left[M^{\prime \prime}, M^{\prime}\right]^{1}-\left[M^{\prime \prime}, M^{\prime}\right]+\sum_{x \in \Delta_{0}} d_{x}^{\prime} d_{x}^{\prime \prime} .
$$

3.3. Let $\mathbf{d} \in \mathbb{N}^{\Delta_{0}}$ and $M \in \bmod _{\Lambda}(\mathbf{d})$. There is a natural inclusion of the tangent space $T_{M} \bmod _{\Lambda}(\mathbf{d})$ to $\bmod _{\Lambda}(\mathbf{d})$ at $M$ into $Z(M, M)($ see $[15,(2.7)])$. If $[M, M]^{2}=0$, then this map is an isomorphism. Indeed, we have a sequence of inequalities, which implies the claim:

$$
\begin{aligned}
a(\mathbf{d}) & =\operatorname{dim} \mathrm{GL}(\mathbf{d})-\langle\mathbf{d}, \mathbf{d}\rangle=\operatorname{dim} \mathrm{GL}(\mathbf{d})-[M, M]+[M, M]^{1} \\
& =\operatorname{dim}_{k} Z(M, M) \geq \operatorname{dim}_{k} T_{M} \bmod _{\Lambda}(\mathbf{d}) \geq \operatorname{dim}_{M} \bmod _{\Lambda}(\mathbf{d}) \geq a(\mathbf{d}),
\end{aligned}
$$

where $\operatorname{dim}_{M} \bmod _{\Lambda}(\mathbf{d})$ denotes the dimension of $\bmod _{\Lambda}(\mathbf{d})$ at $M$, i.e., the maximum of the dimensions of the irreducible components of $\bmod _{\Lambda}(\mathbf{d})$ passing through $M$. It also follows from the above calculations that if $[M, M]^{2}=0$, then $\operatorname{dim}_{M} \bmod _{\Lambda}(\mathbf{d})=$ $a(\mathbf{d})$ and $M$ is a nonsingular point of $\bmod _{\Lambda}(\mathbf{d})$ (see also [12] for a general proof of the last assertion).

Using similar inequalities and the fact that $Z(M, M)$ is the tangent space to the corresponding (not necessarily reduced) scheme (see [23]), one proves the following fact.

Proposition. If $[M, M]^{2}$ vanishes generically on $\bmod _{\Lambda}(\mathbf{d})$, then the variety $\bmod _{\Lambda}(\mathbf{d})$ is a complete intersection of dimension $a(\mathbf{d})$. Moreover, in the above situation $M \in \bmod _{\Lambda}(\mathbf{d})$ is nonsingular if and only if $[M, M]^{2}=0$. 
Proof. This is just a more general formulation of the fact proved in [3, Section 1].

3.4. Let $\mathbf{d}^{\prime}$ and $\mathbf{d}^{\prime \prime}$ be dimension vectors. Put $\mathbf{d}=\mathbf{d}^{\prime}+\mathbf{d}^{\prime \prime}$. Let $C^{\prime}$ be a constructible irreducible $\mathrm{GL}\left(\mathbf{d}^{\prime}\right)$-invariant subset of $\bmod _{\Lambda}\left(\mathbf{d}^{\prime}\right)$ and let $C^{\prime \prime}$ be a constructible irreducible $\operatorname{GL}\left(\mathbf{d}^{\prime \prime}\right)$-invariant subset of $\bmod _{\Lambda}\left(\mathbf{d}^{\prime \prime}\right)$. Let

$$
\begin{aligned}
& \operatorname{hom}\left(C^{\prime}, C^{\prime \prime}\right)=\min \left\{\left[M^{\prime}, M^{\prime \prime}\right] \mid M^{\prime} \in C^{\prime}, M^{\prime \prime} \in C^{\prime \prime}\right\}, \\
& \operatorname{ext}^{1}\left(C^{\prime}, C^{\prime \prime}\right)=\min \left\{\left[M^{\prime}, M^{\prime \prime}\right]^{1} \mid M^{\prime} \in C^{\prime}, M^{\prime \prime} \in C^{\prime \prime}\right\},
\end{aligned}
$$

and

$$
\operatorname{ext}^{2}\left(C^{\prime}, C^{\prime \prime}\right)=\min \left\{\left[M^{\prime}, M^{\prime \prime}\right]^{2} \mid M^{\prime} \in C^{\prime}, M^{\prime \prime} \in C^{\prime \prime}\right\} .
$$

Recall from [8, Lemma 4.3] that the functions

$$
\begin{aligned}
& C^{\prime} \times C^{\prime \prime} \ni\left(M^{\prime}, M^{\prime \prime}\right) \mapsto\left[M^{\prime}, M^{\prime \prime}\right] \in \mathbb{Z}, \\
& C^{\prime} \times C^{\prime \prime} \ni\left(M^{\prime}, M^{\prime \prime}\right) \mapsto\left[M^{\prime}, M^{\prime \prime}\right]^{1} \in \mathbb{Z}
\end{aligned}
$$

are upper semicontinuous. Moreover, in our case

$$
\left[M^{\prime}, M^{\prime \prime}\right]^{2}=\operatorname{dim}_{k} Z\left(M^{\prime}, M^{\prime \prime}\right)-\sum_{\gamma \in \Delta_{1}} d_{s \gamma}^{\prime} d_{t \gamma}^{\prime \prime}+\sum_{\rho \in R} d_{s \rho}^{\prime} d_{t \rho}^{\prime \prime},
$$

hence the function

$$
C^{\prime} \times C^{\prime \prime} \ni\left(M^{\prime}, M^{\prime \prime}\right) \mapsto\left[M^{\prime}, M^{\prime \prime}\right]^{2} \in \mathbb{Z}
$$

is also upper semicontinuous (using standard projective resolutions one may prove this fact in a more general setting). In particular, the sets

$$
\begin{aligned}
& \left\{\left(M^{\prime}, M^{\prime \prime}\right) \in C^{\prime} \times C^{\prime \prime} \mid\left[M^{\prime}, M^{\prime \prime}\right]=\operatorname{hom}\left(C^{\prime}, C^{\prime \prime}\right)\right\}, \\
& \left\{\left(M^{\prime}, M^{\prime \prime}\right) \in C^{\prime} \times C^{\prime \prime} \mid\left[M^{\prime}, M^{\prime \prime}\right]^{1}=\operatorname{ext}^{1}\left(C^{\prime}, C^{\prime \prime}\right)\right\}, \\
& \left\{\left(M^{\prime}, M^{\prime \prime}\right) \in C^{\prime} \times C^{\prime \prime} \mid\left[M^{\prime}, M^{\prime \prime}\right]^{2}=\operatorname{ext}^{2}\left(C^{\prime}, C^{\prime \prime}\right)\right\}
\end{aligned}
$$

are open subsets of $C^{\prime} \times C^{\prime \prime}$.

We define $C^{\prime} \oplus C^{\prime \prime}$ to be the set of all $M \in \bmod _{\Lambda}(\mathbf{d})$ which are isomorphic to a module of the form $M^{\prime} \oplus M^{\prime \prime}$ for $M^{\prime} \in C^{\prime}$ and $M^{\prime \prime} \in C^{\prime \prime}$. We have the following formula for the dimension of $C^{\prime} \oplus C^{\prime \prime}$.

Lemma. If $C^{\prime}$ and $C^{\prime \prime}$ are as above, then $C^{\prime} \oplus C^{\prime \prime}$ is a constructible $\mathrm{GL}(\mathbf{d})$-invariant irreducible subset of $\bmod _{\Lambda}(\mathbf{d})$ of dimension

$$
\begin{aligned}
\operatorname{dim} C^{\prime}+\operatorname{dim} C^{\prime \prime} & +\operatorname{dim} \mathrm{GL}(\mathbf{d}) \\
& -\operatorname{dim} \mathrm{GL}\left(\mathbf{d}^{\prime}\right)-\operatorname{dim} \mathrm{GL}\left(\mathbf{d}^{\prime \prime}\right)-\operatorname{hom}\left(C^{\prime}, C^{\prime \prime}\right)-\operatorname{hom}\left(C^{\prime \prime}, C^{\prime}\right) .
\end{aligned}
$$

Proof. The claim follows by considering the map

$$
\mathrm{GL}(\mathbf{d}) \times C^{\prime} \times C^{\prime \prime} \ni\left(g, M^{\prime}, M^{\prime \prime}\right) \mapsto g \cdot\left(M^{\prime} \oplus M^{\prime \prime}\right) \in \bmod _{\Lambda}(\mathbf{d})
$$

(compare for example [8, Section 1]).

A special case of the above lemma, which is really of interest for us, is the following. 
Corollary. Let $C^{\prime}$ and $C^{\prime \prime}$ be as above. If $\operatorname{dim} C^{\prime}=a\left(\mathbf{d}^{\prime}\right)-c_{1}, \operatorname{dim} C^{\prime \prime}=a\left(\mathbf{d}^{\prime \prime}\right)-c_{2}$, $\operatorname{hom}\left(C^{\prime}, C^{\prime \prime}\right)=\left\langle\mathbf{d}^{\prime}, \mathbf{d}^{\prime \prime}\right\rangle$ and $\operatorname{hom}\left(C^{\prime \prime}, C^{\prime}\right)=0$, then $C^{\prime} \oplus C^{\prime \prime}$ is a constructible irreducible subset of $\bmod _{\Lambda}(\mathbf{d})$ of dimension

$$
a(\mathbf{d})+\left\langle\mathbf{d}^{\prime \prime}, \mathbf{d}^{\prime}\right\rangle-\left(c_{1}+c_{2}\right) .
$$

Proof. Direct calculations.

3.5. Let $C^{\prime}$ and $C^{\prime \prime}$ be as above. By $\mathcal{E}\left(C^{\prime}, C^{\prime \prime}\right)$ we mean the set of all

$$
M \in \bmod _{\Lambda}\left(\mathbf{d}^{\prime}+\mathbf{d}^{\prime \prime}\right)
$$

for which there exists an exact sequence

$$
0 \rightarrow M^{\prime \prime} \rightarrow M \rightarrow M^{\prime} \rightarrow 0
$$

with $M^{\prime} \in C^{\prime}$ and $M^{\prime \prime} \in C^{\prime \prime}$. It follows from [8, Theorem 1.3(i)] that if $C^{\prime}$ and $C^{\prime \prime}$ are closed subsets of $\bmod _{\Lambda}\left(\mathbf{d}^{\prime}\right)$ and $\bmod _{\Lambda}\left(\mathbf{d}^{\prime \prime}\right)$ respectively, then $\mathcal{E}\left(C^{\prime}, C^{\prime \prime}\right)$ is a closed subset of $\bmod _{\Lambda}\left(\mathbf{d}^{\prime}+\mathbf{d}^{\prime \prime}\right)$.

3.6. Let $\mathbf{d}$ be a dimension vector. Let $\bmod _{\Lambda}^{P}$ be the full subcategory of $\Lambda$-modules of projective dimension at most 1. Barot and Schröer proved in [1, Proposition 3.1] that if $\bmod _{\Lambda}^{P}(\mathbf{d})$ is nonempty, then it is an irreducible open subset of $\bmod _{\Lambda}(\mathbf{d})$ of dimension $a(\mathbf{d})$. Here, for a subcategory $\mathcal{X}$ of $\bmod _{\Lambda}$ and $\mathbf{d} \in \mathbb{N}^{\Delta_{0}}$, we denote by $\mathcal{X}(\mathbf{d})$ the set of all $M \in \bmod _{\Lambda}(\mathbf{d})$ such that $M \in \mathcal{X}$. Dually, $\bmod _{\Lambda}^{I}(\mathbf{d})$ (if nonempty) is an irreducible open subset of $\bmod _{\Lambda}(\mathbf{d})$ of dimension $a(\mathbf{d})$, where $\bmod _{\Lambda}^{I}$ is the full subcategory of $\Lambda$-modules of injective dimension at most 1 .

3.7. From now until the end of the section we assume that $\Lambda$ is a fixed canonical algebra. The first observation is the following.

Lemma. If $\mathbf{d} \in \mathbf{P}+\mathbf{R}$, then $(\mathcal{P} \vee \mathcal{R})(\mathbf{d})$ is an open subset of $\bmod _{\Lambda}^{P}(\mathbf{d})$. In particular, $\operatorname{dim}(\mathcal{P} \vee \mathcal{R})(\mathbf{d})=a(\mathbf{d})$.

By duality, if $\mathbf{d} \in \mathbf{R}+\mathbf{Q}$, then $(\mathcal{R} \vee \mathcal{Q})(\mathbf{d})$ is an open subset of $\bmod _{\Lambda}^{I}(\mathbf{d})$ of dimension $a(\mathbf{d})$. As a consequence it also follows that if $\mathbf{d} \in \mathcal{R}$, then $\mathcal{R}(\mathbf{d})=$ $(\mathcal{P} \vee \mathcal{R})(\mathbf{d}) \cap(\mathcal{R} \vee \mathcal{Q})(\mathbf{d})$ is an irreducible open subset of $\bmod _{\Lambda}(\mathbf{d})$ of dimension $a(\mathbf{d})$ (see also [10, Section 4] for another explanation of the last fact).

Proof. We already know that $(\mathcal{P} \vee \mathcal{R})(\mathbf{d})$ is contained in $\bmod _{\Lambda}^{P}(\mathbf{d})$, thus it only remains to show that it is open. But $M \in(\mathcal{P} \vee \mathcal{R})(\mathbf{d})$ if and only if there exists $X \in$ $\mathcal{R}$ of dimension vector $\mathbf{h}$ such that $\operatorname{Hom}_{\Lambda}(X, M)=0$, hence the claim follows.

3.8. The proof of an analogous fact for $\mathbf{d} \in \mathbf{P}$ is more involved.

Lemma. If $\mathbf{d} \in \mathbf{P}$, then $\mathcal{P}(\mathbf{d})$ is an open subset of $\bmod _{\Lambda}^{P}(\mathbf{d})$. In particular, $\operatorname{dim} \mathcal{P}(\mathbf{d})=a(\mathbf{d})$.

By duality, if $\mathbf{d} \in \mathbf{Q}$, then $\mathcal{Q}(\mathbf{d})$ is an open subset of $\bmod _{\Lambda}^{I}(\mathbf{d})$ of dimension $a(\mathbf{d})$.

Proof. Again we only have to show that $\mathcal{P}(\mathbf{d})$ is an open subset of $\bmod _{\Lambda}(\mathbf{d})$, hence also of $\bmod _{\Lambda}^{P}(\mathbf{d})$. We prove that $\mathcal{P}(\mathbf{d})$ is the complement of the sum

$$
\bigcup_{\substack{\mathbf{d}^{\prime} \in \mathbf{P}, \mathbf{d}^{\prime \prime} \in \mathbf{R}+\mathbf{Q} \\ \mathbf{d}^{\prime}+\mathbf{d}^{\prime \prime}=\mathbf{d}, \mathbf{d}^{\prime \prime} \neq 0}} \mathcal{E}\left(\bmod _{\Lambda}\left(\mathbf{d}^{\prime}\right), \bmod _{\Lambda}\left(\mathbf{d}^{\prime \prime}\right)\right) .
$$


Since this is a finite sum of sets which are closed by Subsection 3.5, it will imply the lemma. In order to show the above claim, take $M \notin \mathcal{P}(\mathbf{d})$. Then $M=M^{\prime} \oplus M^{\prime \prime}$ for some $M^{\prime} \in \mathcal{P}$ and $M^{\prime \prime} \in \mathcal{R} \vee \mathcal{Q}, M^{\prime \prime} \neq 0$, and obviously

$$
M \in \mathcal{E}\left(\bmod _{\Lambda}\left(\operatorname{dim} M^{\prime}\right), \bmod _{\Lambda}\left(\operatorname{dim} M^{\prime \prime}\right)\right) .
$$

Assume now that $M \in \mathcal{E}\left(\bmod _{\Lambda}\left(\mathbf{d}^{\prime}\right), \bmod _{\Lambda}\left(\mathbf{d}^{\prime \prime}\right)\right)$ for $\mathbf{d}^{\prime}$ and $\mathbf{d}^{\prime \prime}$ as above. Let

$$
0 \rightarrow M^{\prime \prime} \rightarrow M \rightarrow M^{\prime} \rightarrow 0
$$

be a short exact sequence with $M^{\prime} \in \bmod _{\Lambda}\left(\mathbf{d}^{\prime}\right)$ and $M^{\prime \prime} \in \bmod _{\Lambda}\left(\mathbf{d}^{\prime \prime}\right)$. Since $\left\langle\operatorname{dim} M^{\prime \prime}, \mathbf{h}\right\rangle \leq 0$, it follows that $M^{\prime \prime}$ has a nonzero direct summand $N^{\prime \prime} \in \mathcal{R} \vee \mathcal{Q}$. Since $\operatorname{Hom}_{\Lambda}\left(N^{\prime \prime}, N\right)=0$ for all $N \in \mathcal{P}$, we get $M \notin \mathcal{P}$, and we are done.

\section{Proofs of Theorems 1.1 And 1.2}

4.1. We prove Theorems 1.1 and 1.2 in a more general setting. Let $\Lambda$ be the path algebra of a bound quiver $(\Delta, R)$ of global dimension at most 2 . We also assume that we are given two full subcategories $\mathcal{X}$ and $\mathcal{Y}$ of $\bmod _{\Lambda}$ having the following properties:

(1) $\mathcal{X}$ and $\mathcal{Y}$ are closed under forming direct sums and taking direct summands,

(2) $\mathcal{X} \vee \mathcal{Y}=\bmod _{\Lambda}$

(3) $\operatorname{pd}_{\Lambda} M \leq 1$ for $M \in \mathcal{X}$ and $\operatorname{id}_{\Lambda} N \leq 1$ for $N \in \mathcal{Y}$,

(4) $[N, M]=0$ and $[M, N]^{1}=0$ for $N \in \mathcal{Y}$ and $M \in \mathcal{X}$,

(5) if $\mathbf{d} \in \mathbb{N}^{\Delta_{0}}$, then $\mathcal{X}(\mathbf{d})$ and $\mathcal{Y}(\mathbf{d})$ are open subsets of $\bmod _{\Lambda}(\mathbf{d})$.

Observe that canonical algebras fit into the above setting with $\mathcal{X}=\mathcal{P}$ and $\mathcal{Y}=$ $\mathcal{R} \vee \mathcal{Q}$ (or $\mathcal{X}=\mathcal{P} \vee \mathcal{R}$ and $\mathcal{Y}=\mathcal{Q}$ ).

Let $\mathbf{X}$ and $\mathbf{Y}$ denote the sets of the dimension vectors of the modules belonging to $\mathcal{X}$ and $\mathcal{Y}$, respectively. It follows from the above conditions that if $\mathbf{d} \in \mathbf{X}$ $\left(\mathbf{d} \in \mathbf{Y}\right.$, respectively), then $\mathcal{X}(\mathbf{d})(\mathcal{Y}(\mathbf{d}))$ is an irreducible open subset of $\bmod _{\Lambda}(\mathbf{d})$ of dimension $a(\mathbf{d})$. In particular, if $\mathbf{d}^{\prime} \in \mathbf{X}$ and $\mathbf{d}^{\prime \prime} \in \mathbf{Y}$, then $\mathcal{X}\left(\mathbf{d}^{\prime}\right) \oplus \mathcal{Y}\left(\mathbf{d}^{\prime \prime}\right)$ is an irreducible constructible subset (in fact, using [8, Theorem 1.3(iii)] one can even show that this set is locally closed) of $\bmod _{\Lambda}\left(\mathbf{d}^{\prime}+\mathbf{d}^{\prime \prime}\right)$ of dimension $a\left(\mathbf{d}^{\prime}+\mathbf{d}^{\prime \prime}\right)+$ $\left\langle\mathbf{d}^{\prime \prime}, \mathbf{d}^{\prime}\right\rangle$ (apply Corollary 3.4). Consequently, for $\mathbf{d} \in \mathbb{N}^{\Delta_{0}}, \bmod _{\Lambda}(\mathbf{d})$ is a finite disjoint union

$$
\bigcup_{\substack{\mathbf{d}^{\prime} \in \mathbf{X}, \mathbf{d}^{\prime \prime} \in \mathbf{Y} \\ \mathbf{d}^{\prime}+\mathbf{d}^{\prime \prime}=\mathbf{d}}} \mathcal{X}\left(\mathbf{d}^{\prime}\right) \oplus \mathcal{Y}\left(\mathbf{d}^{\prime \prime}\right)
$$

of irreducible constructible subsets of dimensions $a(\mathbf{d})+\left\langle\mathbf{d}^{\prime \prime}, \mathbf{d}^{\prime}\right\rangle$, respectively. In particular, this implies that

$$
\operatorname{dim} \bmod _{\Lambda}(\mathbf{d})=a(\mathbf{d})+\max \left\{\left\langle\mathbf{d}^{\prime \prime}, \mathbf{d}^{\prime}\right\rangle \mid \mathbf{d}^{\prime} \in \mathbf{X}, \mathbf{d}^{\prime \prime} \in \mathbf{Y}, \mathbf{d}^{\prime}+\mathbf{d}^{\prime \prime}=\mathbf{d}\right\} .
$$

Consequently, $\operatorname{dim} \bmod _{\Lambda}(\mathbf{d})=a(\mathbf{d})$ if and only if $\left\langle\mathbf{d}^{\prime \prime}, \mathbf{d}^{\prime}\right\rangle \leq 0$ for all $\mathbf{d}^{\prime}$ and $\mathbf{d}^{\prime \prime}$ as above (recall that obviously $\operatorname{dim} \bmod _{\Lambda}(\mathbf{d}) \geq a(\mathbf{d})$ ).

4.2. As a first step in proving Theorem 1.2 we prove the following lemma.

Lemma. Let $\mathbf{d} \in \mathbb{N}^{\Delta_{0}}$. If $\left\langle\mathbf{d}^{\prime \prime}, \mathbf{d}^{\prime}\right\rangle \leq 0$ for all $\mathbf{d}^{\prime} \in \mathbf{X}$ and $\mathbf{d}^{\prime \prime} \in \mathbf{Y}$ such that $\mathbf{d}=\mathbf{d}^{\prime}+\mathbf{d}^{\prime \prime}$, then $\bmod _{\Lambda}(\mathbf{d})$ is a complete intersection.

Proof. According to Proposition 3.3, in order to prove that $\bmod _{\Lambda}(\mathbf{d})$ is a complete intersection, it is enough to prove that $[M, M]^{2}$ vanishes generically on $\bmod _{\Lambda}(\mathbf{d})$. Note that every irreducible component of $\bmod _{\Lambda}(\mathbf{d})$ is the closure of the set of the 
form $\mathcal{X}\left(\mathbf{d}^{\prime}\right) \oplus \mathcal{Y}\left(\mathbf{d}^{\prime \prime}\right)$ for some $\mathbf{d}^{\prime} \in \mathbf{X}$ and $\mathbf{d}^{\prime \prime} \in \mathbf{Y}$, such that $\left\langle\mathbf{d}^{\prime \prime}, \mathbf{d}^{\prime}\right\rangle=0$. It is well-known that if the closure of $\mathcal{X}\left(\mathbf{d}^{\prime}\right) \oplus \mathcal{Y}\left(\mathbf{d}^{\prime \prime}\right)$ is an irreducible component of $\bmod _{\Lambda}(\mathbf{d})$, then $\operatorname{ext}^{1}\left(\mathcal{Y}\left(\mathbf{d}^{\prime \prime}\right), \mathcal{X}\left(\mathbf{d}^{\prime}\right)\right)=0$ (see for example [8, Theorem 1.2]). Since obviously $\operatorname{hom}\left(\mathcal{Y}\left(\mathbf{d}^{\prime \prime}\right), \mathcal{X}\left(\mathbf{d}^{\prime}\right)\right)=0$, we get $\operatorname{ext}^{2}\left(\mathcal{Y}\left(\mathbf{d}^{\prime \prime}\right), \mathcal{X}\left(\mathbf{d}^{\prime}\right)\right)=0$ and the claim follows, because $\operatorname{pd}_{\Lambda} M^{\prime} \leq 1$ for $M^{\prime} \in \mathcal{X}$ and $\operatorname{id}_{\Lambda} M^{\prime \prime} \leq 1$ for $M^{\prime \prime} \in \mathcal{Y}$.

4.3. In order to reverse the above implication and finish the proof of Theorem 1.2 we need an additional assumption.

Proposition. Let $\mathbf{d}$ be the dimension vector of a $\Lambda$-module of projective or injective dimension at most 1 . Then $\bmod _{\Lambda}(\mathbf{d})$ is a complete intersection if and only if $\left\langle\mathbf{d}^{\prime \prime}, \mathbf{d}^{\prime}\right\rangle \leq 0$ for all $\mathbf{d}^{\prime} \in \mathbf{X}$ and $\mathbf{d}^{\prime \prime} \in \mathbf{Y}$ such that $\mathbf{d}=\mathbf{d}^{\prime}+\mathbf{d}^{\prime \prime}$.

Proof. We only have to prove that if $\bmod _{\Lambda}(\mathbf{d})$ is a complete intersection, then $\operatorname{dim} \bmod _{\Lambda}(\mathbf{d})=a(\mathbf{d})$, but this follows since $\bmod _{\Lambda}(\mathbf{d})$ has an irreducible component of dimension $a(\mathbf{d})$ (the closure of $\bmod _{\Lambda}^{P}(\mathbf{d})$ or $\left.\bmod _{\Lambda}^{I}(\mathbf{d})\right)$ and complete intersections are equidimensional.

4.4. We also divide the proof of an analogous criterion for irreducibility in two steps.

Lemma. Let $\mathbf{d}$ be the dimension vector. If $\left\langle\mathbf{d}^{\prime \prime}, \mathbf{d}^{\prime}\right\rangle \leq 0$ for all $\mathbf{d}^{\prime} \in \mathbf{X}$ and $\mathbf{d}^{\prime \prime} \in \mathbf{Y}$ such that $\mathbf{d}=\mathbf{d}^{\prime}+\mathbf{d}^{\prime \prime}$, and equality holds for exactly one pair $\left(\mathbf{d}^{\prime}, \mathbf{d}^{\prime \prime}\right)$, then $\bmod _{\Lambda}(\mathbf{d})$ is irreducible.

Proof. Let $\hat{\mathbf{d}}^{\prime} \in \mathbf{X}$ and $\hat{\mathbf{d}}^{\prime \prime} \in \mathbf{Y}$ be such that $\hat{\mathbf{d}}^{\prime}+\hat{\mathbf{d}}^{\prime \prime}=\mathbf{d}$ and $\left\langle\hat{\mathbf{d}}^{\prime \prime}, \hat{\mathbf{d}}^{\prime}\right\rangle=0$. Then $\mathcal{X}\left(\hat{\mathbf{d}}^{\prime}\right) \oplus \mathcal{Y}\left(\hat{\mathbf{d}}^{\prime \prime}\right)$ is an irreducible constructible subset of $\bmod _{\Lambda}(\mathbf{d})$ of dimension $a(\mathbf{d})$, and the remaining sets $\mathcal{X}\left(\mathbf{d}^{\prime}\right) \oplus \mathcal{Y}\left(\mathbf{d}^{\prime \prime}\right)$ have dimensions smaller than $a(\mathbf{d})$. Since every irreducible component of $\bmod _{\Lambda}(\mathbf{d})$ has dimension at least $a(\mathbf{d}), \bmod _{\Lambda}(\mathbf{d})$ is the closure of $\mathcal{X}\left(\hat{\mathbf{d}}^{\prime}\right) \oplus \mathcal{Y}\left(\hat{\mathbf{d}}^{\prime \prime}\right)$, hence irreducible.

4.5. We may again reverse the above implication if we assume the existence of a $\Lambda$-module of dimension vector $\mathbf{d}$ and projective or injective dimension at most 1 .

Proposition. Let $\mathbf{d}$ be the dimension vector of a $\Lambda$-module of projective or injective dimension at most 1 . Then $\bmod _{\Lambda}(\mathbf{d})$ is irreducible if and only if $\left\langle\mathbf{d}^{\prime \prime}, \mathbf{d}^{\prime}\right\rangle \leq 0$ for all $\mathbf{d}^{\prime} \in \mathbf{X}$ and $\mathbf{d}^{\prime \prime} \in \mathbf{Y}$ such that $\mathbf{d}=\mathbf{d}^{\prime}+\mathbf{d}^{\prime \prime}$, and equality holds for exactly one pair $\left(\mathbf{d}^{\prime}, \mathbf{d}^{\prime \prime}\right)$.

Proof. We only have to prove that if $\bmod _{\Lambda}(\mathbf{d})$ is irreducible, then the above condition is satisfied. Without loss of generality we may assume that $\mathbf{d}$ is the dimension vector of a $\Lambda$-module of projective dimension at most 1 . Then we know that the closure of $\bmod _{\Lambda}^{P}(\mathbf{d})$ is an irreducible component of $\bmod _{\Lambda}(\mathbf{d})$ of dimension $a(\mathbf{d})$, thus $\operatorname{dim} \bmod _{\Lambda}(\mathbf{d})=a(\mathbf{d})$. In particular, $\left\langle\mathbf{d}^{\prime \prime}, \mathbf{d}^{\prime}\right\rangle \leq 0$ for all $\mathbf{d}^{\prime}$ and $\mathbf{d}^{\prime \prime}$. Moreover, the irreducible components of $\bmod _{\Lambda}(\mathbf{d})$ are precisely the closures of the sets $\mathcal{X}\left(\mathbf{d}^{\prime}\right) \oplus \mathcal{Y}\left(\mathbf{d}^{\prime \prime}\right)$ with $\operatorname{dim}\left(\mathcal{X}\left(\mathbf{d}^{\prime}\right) \oplus \mathcal{Y}\left(\mathbf{d}^{\prime \prime}\right)\right)=a(\mathbf{d})$, i.e., $\left\langle\mathbf{d}^{\prime \prime}, \mathbf{d}^{\prime}\right\rangle=0$. Thus irreducibility of $\bmod _{\Lambda}(\mathbf{d})$ implies that the equality holds for exactly one pair $\left(\mathbf{d}^{\prime}, \mathbf{d}^{\prime \prime}\right)$.

4.6. As a consequence of the above propositions we also obtain some information about the maximal GL(d)-orbits in the above situations. Namely, if $\mathbf{d}$ is a dimension vector such that $\operatorname{dim} \bmod _{\Lambda}(\mathbf{d})=a(\mathbf{d})$, then every maximal $G L(\mathbf{d})$-orbit consists of points which are nonsingular in $\bmod _{\Lambda}(\mathbf{d})$. Indeed, let the $\mathrm{GL}(\mathbf{d})$-orbit of a $\Lambda$-module $M$ be maximal and write $M=M^{\prime} \oplus M^{\prime \prime}$ for $M^{\prime} \in \mathcal{X}$ and $M^{\prime \prime} \in \mathcal{Y}$. 
We know that $\left\langle\operatorname{dim} M^{\prime \prime}, \operatorname{dim} M^{\prime}\right\rangle \leq 0$. Obviously, $\left[M^{\prime \prime}, M^{\prime}\right]=0$. Moreover, the maximality of the $\mathrm{GL}(\mathbf{d})$-orbit of $M$ implies that $\left[M^{\prime \prime}, M^{\prime}\right]^{1}=0$ (see for example $[6$, Lemma 1.1]). Consequently, $\left[M^{\prime \prime}, M^{\prime}\right]^{2}=0$. Since $\operatorname{pd}_{\Lambda} M^{\prime} \leq 1$ and $\operatorname{id}_{\Lambda} M^{\prime \prime} \leq 1$, this implies that $[M, M]^{2}=0$, which finishes the proof according to Subsection 3.3.

Now assume in addition that $\Lambda$ is a canonical algebra, $\mathbf{d} \in \mathbf{R}$ and $\bmod _{\Lambda}(\mathbf{d})$ is irreducible. Then it follows from Proposition 4.5 that $\left\langle\mathbf{d}^{\prime \prime}, \mathbf{d}^{\prime}\right\rangle<0$ for all $\mathbf{d}^{\prime} \in \mathbf{P}$ and $\mathbf{d}^{\prime \prime} \in \mathbf{R}+\mathbf{Q}$ with $\mathbf{d}^{\prime} \neq 0$. Consequently, again using [6, Lemma 1.1] we obtain that if the GL(d)-orbit of $M$ is maximal, then $M \in \mathcal{R} \vee \mathcal{Q}$. Since $\langle\mathbf{h}, \mathbf{d}\rangle=$ $0,(\mathcal{R} \vee \mathcal{Q})(\mathbf{d})=\mathcal{R}(\mathbf{d})$ and $M \in \mathcal{R}$. With methods analogous to those used in the proofs of [19, Theorem 3.5] and [3, Proposition 5], one can give a precise description of the maximal $\mathrm{GL}(\mathbf{d})$-orbits. It is essentially identical to that given in [3, Proposition 5], but since it is lengthy and requires introducing an appropriate language, we will not present it here.

4.7. We now give the proof of Theorem 1.1. The crucial observation, whose proof is based on ideas of the proof of [18, Proposition 2.5], is the following.

Lemma. Let $\mathbf{d}^{\prime} \in \mathbf{X}$ and $\mathbf{d}^{\prime \prime} \in \mathbf{Y}$. If $\operatorname{dim} \bmod _{\Lambda}\left(\mathbf{d}^{\prime}+\mathbf{d}^{\prime \prime}\right)=a\left(\mathbf{d}^{\prime}+\mathbf{d}^{\prime \prime}\right)$, then $\operatorname{ext}^{1}\left(\mathcal{Y}\left(\mathbf{d}^{\prime \prime}\right), \mathcal{X}\left(\mathbf{d}^{\prime}\right)\right)=-\left\langle\mathbf{d}^{\prime \prime}, \mathbf{d}^{\prime}\right\rangle$.

Proof. Let $\mathbf{d}=\mathbf{d}^{\prime}+\mathbf{d}^{\prime \prime}, C^{\prime}=\mathcal{X}\left(\mathbf{d}^{\prime}\right)$ and $C^{\prime \prime}=\mathcal{Y}\left(\mathbf{d}^{\prime \prime}\right)$. Obviously, ext ${ }^{1}\left(C^{\prime \prime}, C^{\prime}\right) \geq$ $-\left\langle\mathbf{d}^{\prime \prime}, \mathbf{d}^{\prime}\right\rangle$, thus we only have to show that $\operatorname{ext}^{1}\left(C^{\prime \prime}, C^{\prime}\right) \leq-\left\langle\mathbf{d}^{\prime \prime}, \mathbf{d}^{\prime}\right\rangle$.

Recall that $\mathcal{U}=\left\{\left(M^{\prime}, M^{\prime \prime}\right) \in C^{\prime} \times C^{\prime \prime} \mid\left[M^{\prime \prime}, M^{\prime}\right]^{1}=\operatorname{ext}^{1}\left(C^{\prime \prime}, C^{\prime}\right)\right\}$ is an open subset of $C^{\prime} \times C^{\prime \prime}$. Consequently, according to [8, Theorem 1.3(iii)], the subset $\mathcal{V}$ of all $M \in \bmod _{\Lambda}(\mathbf{d})$ such that there exists a short exact sequence

$$
0 \rightarrow M^{\prime} \rightarrow M \rightarrow M^{\prime \prime} \rightarrow 0
$$

with $\left(M^{\prime}, M^{\prime \prime}\right) \in \mathcal{U}$ is an open subset of $\bmod _{\Lambda}(\mathbf{d})$. In particular, $\mathcal{V}$ contains nonsingular points of $\bmod _{\Lambda}(\mathbf{d})$.

Let $\mathcal{Z}=\left\{\left(M^{\prime}, M^{\prime \prime}, Z\right) \mid\left(M^{\prime}, M^{\prime \prime}\right) \in \mathcal{U}, Z \in Z\left(M^{\prime \prime}, M^{\prime}\right)\right\}$. It follows from [5, Lemma 1] that the canonical projection $\mathcal{Z} \rightarrow \mathcal{U}$ is a subbundle of the trivial vector bundle $\mathcal{U} \times \mathbb{A}\left(\mathbf{d}^{\prime}, \mathbf{d}^{\prime \prime}\right) \rightarrow \mathcal{U}$. In particular, $\mathcal{Z}$ is smooth, since $\mathcal{U}$ is an open subset of $\bmod _{\Lambda}\left(\mathbf{d}^{\prime}\right) \times \bmod _{\Lambda}\left(\mathbf{d}^{\prime \prime}\right)$ consisting of nonsingular points. We now describe the tangent space $T_{\left(M^{\prime}, M^{\prime \prime}, Z\right)} \mathcal{Z}$ for $\left(M^{\prime}, M^{\prime \prime}, Z\right) \in \mathcal{Z}$ more precisely. Obviously, it is a subspace of

$$
\mathbb{A}\left(\mathbf{d}^{\prime}, \mathbf{d}^{\prime}\right) \times \mathbb{A}\left(\mathbf{d}^{\prime \prime}, \mathbf{d}^{\prime \prime}\right) \times \mathbb{A}\left(\mathbf{d}^{\prime}, \mathbf{d}^{\prime \prime}\right)
$$

of dimension

$$
a\left(\mathbf{d}^{\prime}\right)+a\left(\mathbf{d}^{\prime \prime}\right)+\operatorname{ext}^{1}\left(C^{\prime \prime}, C^{\prime}\right)+\sum_{x \in \Delta_{0}} d_{x}^{\prime} d_{x}^{\prime \prime}
$$

Moreover, if $\left(Z^{\prime}, Z^{\prime \prime}, Y\right) \in T_{\left(M^{\prime}, M^{\prime \prime}, Z\right)} \mathcal{Z}$, then $Z^{\prime} \in Z\left(M^{\prime}, M^{\prime}\right), Z^{\prime \prime} \in Z\left(M^{\prime \prime}, M^{\prime \prime}\right)$ and $Y_{\rho}=r_{\rho}$ for all $\rho \in R$, where $Y_{\rho}$ is defined as in Subsection 3.3 and we define $r_{\rho}$ by the standard extension to relations of the following definition for paths: if $\sigma=\gamma_{1} \cdots \gamma_{m}$ is a path of length at least 2 , then

$$
\begin{aligned}
r_{\sigma}=\sum_{i<j \in[1, m]} M_{\gamma_{1}}^{\prime} \cdots M_{\gamma_{i-1}}^{\prime} Z_{\gamma_{i}}^{\prime} M_{\gamma_{i+1}}^{\prime} \cdots M_{\gamma_{j-1}}^{\prime} Z_{\gamma_{j}} M_{\gamma_{j+1}}^{\prime \prime} \cdots M_{\gamma_{m}}^{\prime \prime} \\
+\sum_{i<j \in[1, m]} M_{\gamma_{1}}^{\prime} \cdots M_{\gamma_{i-1}}^{\prime} Z_{\gamma_{i}} M_{\gamma_{i+1}}^{\prime \prime} \cdots M_{\gamma_{j-1}}^{\prime \prime} Z_{\gamma_{j}}^{\prime \prime} M_{\gamma_{j+1}}^{\prime \prime} \cdots M_{\gamma_{m}}^{\prime \prime}
\end{aligned}
$$


Since $\operatorname{dim}_{k} Z\left(M^{\prime}, M^{\prime}\right)=a\left(\mathbf{d}^{\prime}\right), \operatorname{dim}_{k} Z\left(M^{\prime \prime}, M^{\prime \prime}\right)=a\left(\mathbf{d}^{\prime \prime}\right)$ and the solution set of the homogeneous system $Y_{\rho}=0, \rho \in R$, is $Z\left(M^{\prime \prime}, M^{\prime}\right)$, it follows by comparing dimensions that $T_{\left(M^{\prime}, M^{\prime \prime}, Z\right)} \mathcal{Z}$ is the set of all $\left(Z^{\prime}, Z^{\prime \prime}, Y\right)$ satisfying the above conditions.

Note that $T_{\mathrm{Id}} \mathrm{GL}(\mathbf{d})=\mathbb{V}(\mathbf{d}, \mathbf{d})$ (see Subsection 3.2 for a definition). With respect to the canonical decomposition

$$
\mathbb{V}(\mathbf{d}, \mathbf{d})=\left[\begin{array}{rr}
\mathbb{V}\left(\mathbf{d}^{\prime}, \mathbf{d}^{\prime}\right) & \mathbb{V}\left(\mathbf{d}^{\prime}, \mathbf{d}^{\prime \prime}\right) \\
\mathbb{V}\left(\mathbf{d}^{\prime \prime}, \mathbf{d}^{\prime}\right) & \mathbb{V}\left(\mathbf{d}^{\prime \prime}, \mathbf{d}^{\prime \prime}\right)
\end{array}\right]
$$

every element $X \in \mathbb{V}(\mathbf{d}, \mathbf{d})$ can be written as

$$
X=\left[\begin{array}{ll}
X^{(1,1)} & X^{(1,2)} \\
X^{(2,1)} & X^{(2,2)}
\end{array}\right] .
$$

On the other hand, if $M \in \mathcal{V}$, then $T_{M} \mathcal{V}=T_{M} \bmod _{\Lambda}(\mathbf{d}) \subset \mathbb{A}(\mathbf{d}, \mathbf{d})$, and the canonical decomposition

$$
\mathbb{A}(\mathbf{d}, \mathbf{d})=\left[\begin{array}{ll}
\mathbb{A}\left(\mathbf{d}^{\prime}, \mathbf{d}^{\prime}\right) & \mathbb{A}\left(\mathbf{d}^{\prime}, \mathbf{d}^{\prime \prime}\right) \\
\mathbb{A}\left(\mathbf{d}^{\prime \prime}, \mathbf{d}^{\prime}\right) & \mathbb{A}\left(\mathbf{d}^{\prime \prime}, \mathbf{d}^{\prime \prime}\right)
\end{array}\right]
$$

induces the analogous matrix presentation of the elements of $T_{M} \mathcal{V}$.

We have a surjective map $\Phi: \operatorname{GL}(\mathbf{d}) \times \mathcal{Z} \rightarrow \mathcal{V}$ given by

$$
\left(g, M^{\prime}, M^{\prime \prime}, Z\right) \mapsto g \cdot M, \text { where } M_{\gamma}=\left[\begin{array}{cc}
M_{\gamma}^{\prime} & Z_{\gamma} \\
0 & M_{\gamma}^{\prime \prime}
\end{array}\right], \gamma \in \Delta_{1} .
$$

For fixed $\left(M^{\prime}, M^{\prime \prime}, Z\right) \in \mathcal{Z}$, we have the tangent map $F: T_{\mathrm{Id}} \mathrm{GL}(\mathbf{d}) \times T_{\left(M^{\prime}, M^{\prime \prime}, Z\right)} \mathcal{Z} \rightarrow$ $T_{M} \mathcal{V}$ given by (here we apply the conventions for presenting the elements of $\mathbb{V}(\mathbf{d}, \mathbf{d})$ and $T_{M} \mathcal{V}$ introduced above)

$$
\begin{aligned}
F\left(X, Z^{\prime}, Z^{\prime \prime}, Y\right)_{\gamma}^{(1,1)}= & Z_{\gamma}^{\prime}+X_{t \gamma}^{(1,1)} M_{\gamma}^{\prime}-M_{\gamma}^{\prime} X_{s \gamma}^{(1,1)}-Z_{\gamma} X_{s \gamma}^{(2,1)} \\
F\left(X, Z^{\prime}, Z^{\prime \prime}, Y\right)_{\gamma}^{(1,2)}= & Y_{\gamma}+X_{t \gamma}^{(1,1)} Z_{\gamma}+X_{t \gamma}^{(1,2)} M_{\gamma}^{\prime \prime} \\
& \quad-M_{\gamma}^{\prime} X_{s \gamma}^{(1,2)}-Z_{\gamma} X_{s \gamma}^{(2,2)} \\
F\left(X, Z^{\prime}, Z^{\prime \prime}, Y\right)_{\gamma}^{(2,1)}= & X_{t \gamma}^{(2,1)} M_{\gamma}^{\prime}-M_{\gamma}^{\prime \prime} X_{s \gamma}^{(2,1)}
\end{aligned}
$$

and

$$
F\left(X, Z^{\prime}, Z^{\prime \prime}, Y\right)_{\gamma}^{(2,2)}=Z_{\gamma}^{\prime \prime}+X_{t \gamma}^{(2,1)} Z_{\gamma}+X_{t \gamma}^{(2,2)} M_{\gamma}^{\prime \prime}-M_{\gamma}^{\prime \prime} X_{s \gamma}^{(2,2)},
$$

for $\gamma \in \Delta_{1}$ (it follows by computations using block matrices - compare the corresponding calculations in the proof of [18, Proposition 2.5]).

Note that $X^{(2,1)} \in \operatorname{Hom}_{\Lambda}\left(M^{\prime}, M^{\prime \prime}\right)$ for $\left(X, Z^{\prime}, Z^{\prime \prime}, Y\right) \in \operatorname{Ker} F$. Thus the linear map $G: \operatorname{Ker} F \rightarrow \operatorname{Hom}_{\Lambda}\left(M^{\prime}, M^{\prime \prime}\right)$ given by $G\left(X, Z^{\prime}, Z^{\prime \prime}, Y\right)=X^{(2,1)}$ is welldefined. Moreover, $G$ is surjective. Indeed, for $f: M^{\prime} \rightarrow M^{\prime \prime}$ we define

$$
\begin{aligned}
X & =\left[\begin{array}{ll}
0 & 0 \\
f & 0
\end{array}\right], & Y_{\gamma} & =0, \\
Z_{\gamma}^{\prime} & =Z_{\gamma} f_{s \gamma}, & Z_{\gamma}^{\prime \prime} & =-f_{t \gamma} Z_{\gamma},
\end{aligned}
$$

where $\gamma \in \Delta_{1}$. One checks that $\left(Z^{\prime}, Z^{\prime \prime}, Y\right) \in T_{\left(M^{\prime}, M^{\prime \prime}, Z\right)} \mathcal{Z}$. Moreover,

$$
F\left(X, Z^{\prime}, Z^{\prime \prime}, Y\right)=0 \text { and } G\left(X, Z^{\prime}, Z^{\prime \prime}, Y\right)=f \text {. }
$$


Let $\mathfrak{p}=\left\{X \in \mathbb{V}(\mathbf{d}) \mid X^{(2,1)}=0\right\}$. Define $H: \mathfrak{p} \rightarrow T_{\mathrm{Id}} \mathrm{GL}(\mathbf{d}) \times T_{\left(M^{\prime}, M^{\prime \prime}, Z\right)} \mathcal{Z}$ by $H(X)=\left(X, Z^{\prime}, Z^{\prime \prime}, Y\right)$, where

$$
\begin{aligned}
& Z_{\gamma}^{\prime}=M_{\gamma}^{\prime} X_{s \gamma}^{(1,1)}-X_{t \gamma}^{(1,1)} M_{\gamma}^{\prime}, \\
& Z_{\gamma}^{\prime \prime}=M_{\gamma}^{\prime \prime} X_{s \gamma}^{(2,2)}-X_{t \gamma}^{(2,2)} M_{\gamma}^{\prime \prime},
\end{aligned}
$$

and

$$
Y_{\gamma}=M_{\gamma}^{\prime} X_{s \gamma}^{(1,2)}+Z_{\gamma} X_{s \gamma}^{(2,2)}-X_{t \gamma}^{(1,1)} Z_{\gamma}-X_{t \gamma}^{(1,2)} M_{\gamma}^{\prime \prime},
$$

for $\gamma \in \Delta_{1}$. Using the description of $T_{\left(M^{\prime}, M^{\prime \prime}, Z\right)} \mathcal{Z}$ one checks that $H$ is welldefined. Obviously, $H$ is injective. Moreover, by direct calculations one checks that $\operatorname{Im} H=\operatorname{Ker} G$. Consequently, we get

$\operatorname{dim}_{k} \operatorname{Ker} F=\operatorname{dim}_{k} \mathfrak{p}+\operatorname{dim}_{k} \operatorname{Hom}_{\Lambda}\left(M^{\prime}, M^{\prime \prime}\right)$

$$
=\operatorname{dim} \mathrm{GL}\left(\mathbf{d}^{\prime}\right)+\operatorname{dim} \mathrm{GL}\left(\mathbf{d}^{\prime \prime}\right)+\sum_{x \in \Delta_{0}} d_{x}^{\prime} d_{x}^{\prime \prime}+\left\langle\mathbf{d}^{\prime}, \mathbf{d}^{\prime \prime}\right\rangle .
$$

We may assume that $M$ is a nonsingular point of $\bmod _{\Lambda}(\mathbf{d})$. Then we have the following sequence of inequalities:

$$
\begin{aligned}
a(\mathbf{d})= & \operatorname{dim}_{M} \bmod _{\Lambda}(\mathbf{d})=\operatorname{dim}_{k} T_{M} \bmod _{\Lambda}(\mathbf{d})=\operatorname{dim}_{k} T_{M} \mathcal{V} \\
\geq & \operatorname{dim}_{k} \operatorname{Im} F=\operatorname{dim}_{k} \mathbb{V}(\mathbf{d})+\operatorname{dim}_{k} T_{\left(M^{\prime}, M^{\prime \prime}, Z\right)} \mathcal{Z}-\operatorname{dim}_{k} \operatorname{Ker} F \\
= & \operatorname{dim} \operatorname{GL}(\mathbf{d})+a\left(\mathbf{d}^{\prime}\right)+a\left(\mathbf{d}^{\prime \prime}\right)+\operatorname{ext}^{1}\left(C^{\prime \prime}, C^{\prime}\right)+\sum_{x \in \Delta_{0}} d_{x}^{\prime} d_{x}^{\prime \prime} \\
& \quad-\operatorname{dim} \operatorname{GL}\left(\mathbf{d}^{\prime}\right)-\operatorname{dim} \operatorname{GL}\left(\mathbf{d}^{\prime \prime}\right)-\sum_{x \in \Delta_{0}} d_{x}^{\prime} d_{x}^{\prime \prime}-\left\langle\mathbf{d}^{\prime}, \mathbf{d}^{\prime \prime}\right\rangle \\
= & a(\mathbf{d})+\left\langle\mathbf{d}^{\prime \prime}, \mathbf{d}^{\prime}\right\rangle+\operatorname{ext}^{1}\left(C^{\prime \prime}, C^{\prime}\right),
\end{aligned}
$$

which implies $\operatorname{ext}^{1}\left(C^{\prime \prime}, C^{\prime}\right) \leq-\left\langle\mathbf{d}^{\prime \prime}, \mathbf{d}^{\prime}\right\rangle$, hence finishes the proof.

4.8. Another useful observation is the following.

Lemma. Let $\mathbf{d}$ be a dimension vector such that $\bmod _{\Lambda}(\mathbf{d})$ is irreducible, $\bmod _{\Lambda}^{P}(\mathbf{d}) \neq$ $\varnothing$ and $\bmod _{\Lambda}^{I}(\mathbf{d}) \neq \varnothing$. If $\mathbf{d}^{\prime} \in \mathbf{X}$ and $\mathbf{d}^{\prime \prime} \in \mathbf{Y}$ are such that $\mathbf{d}^{\prime}+\mathbf{d}^{\prime \prime}=\mathbf{d}$ and $\left\langle\mathbf{d}^{\prime \prime}, \mathbf{d}^{\prime}\right\rangle=0$, then the set of $M \in \mathcal{X}\left(\mathbf{d}^{\prime}\right) \oplus \mathcal{Y}\left(\mathbf{d}^{\prime \prime}\right)$ such that $[M, M]^{2} \neq 0$ has dimension at most $a(\mathbf{d})-2$.

Proof. Our assumptions imply that $\bmod _{\Lambda}(\mathbf{d})$ is the closure of $\mathcal{X}\left(\mathbf{d}^{\prime}\right) \oplus \mathcal{Y}\left(\mathbf{d}^{\prime \prime}\right)$, hence $\left(\mathcal{X}\left(\mathbf{d}^{\prime}\right) \oplus \mathcal{Y}\left(\mathbf{d}^{\prime \prime}\right)\right) \cap \bmod _{\Lambda}^{P}(\mathbf{d}) \neq \varnothing$ and $\left(\mathcal{X}\left(\mathbf{d}^{\prime}\right) \oplus \mathcal{Y}\left(\mathbf{d}^{\prime \prime}\right)\right) \cap \bmod _{\Lambda}^{I}(\mathbf{d}) \neq \varnothing$. In particular $\mathcal{X}\left(\mathbf{d}^{\prime}\right) \cap \bmod _{\Lambda}^{I}\left(\mathbf{d}^{\prime}\right) \neq \varnothing$ and $\mathcal{Y}\left(\mathbf{d}^{\prime \prime}\right) \cap \bmod _{\Lambda}^{P}\left(\mathbf{d}^{\prime \prime}\right) \neq \varnothing$. Consequently, $\operatorname{dim} C^{\prime} \leq$ $a\left(\mathbf{d}^{\prime}\right)-1$ and $\operatorname{dim} C^{\prime \prime} \leq a\left(\mathbf{d}^{\prime \prime}\right)-1$, where $C^{\prime}=\left\{M^{\prime} \in \mathcal{X}\left(\mathbf{d}^{\prime}\right) \mid \operatorname{id}_{\Lambda} M^{\prime}=2\right\}$ and $C^{\prime \prime}=\left\{M^{\prime \prime} \in \mathcal{Y}\left(\mathbf{d}^{\prime \prime}\right) \mid \operatorname{pd}_{\Lambda} M^{\prime \prime}=2\right\}$. Since $\left\{M \in \mathcal{X}\left(\mathbf{d}^{\prime}\right) \oplus \mathcal{Y}\left(\mathbf{d}^{\prime \prime}\right) \mid[M, M]^{2} \neq 0\right\} \subset$ $C^{\prime} \oplus C^{\prime \prime}$, the claim follows from Corollary 3.4.

4.9. The following fact implies Theorem 1.1.

Proposition. Let $\mathbf{d}$ be a dimension vector such that at least one of the following conditions is satisfied:

(1) $\mathbf{d} \in \mathbf{X}$ or $\mathbf{d} \in \mathbf{Y}$,

(2) $\bmod _{\Lambda}^{P}(\mathbf{d}) \neq \varnothing$ and $\bmod _{\Lambda}^{I}(\mathbf{d}) \neq \varnothing$.

Then $\bmod _{\Lambda}(\mathbf{d})$ is normal if and only if it is irreducible. 
Proof. We only have to prove that if $\bmod _{\Lambda}(\mathbf{d})$ is irreducible, then it is normal. First observe that irreducibility of $\bmod _{\Lambda}(\mathbf{d})$ implies that either $\bmod _{\Lambda}^{P}(\mathbf{d}) \operatorname{or}_{\bmod _{\Lambda}}^{I}(\mathbf{d})$ is a dense open subset of $\bmod _{\Lambda}(\mathbf{d})$. In particular, $[M, M]^{2}$ vanishes generically on $\bmod _{\Lambda}(\mathbf{d})$, hence $\bmod _{\Lambda}(\mathbf{d})$ is a complete intersection by Proposition 3.3. Consequently, according to Serre's criterion, in order to prove normality we have to show that $\bmod _{\Lambda}(\mathbf{d})$ is nonsingular in codimension 1. According to Proposition 3.3, this will follow if we show that the set of $M \in \bmod _{\Lambda}(\mathbf{d})$ such that $[M, M]^{2} \neq 0$ is of codimension at least 2 .

Recall that

$$
\bmod _{\Lambda}(\mathbf{d})=\bigcup_{\substack{\mathbf{d}^{\prime} \in \mathbf{X}, \mathbf{d}^{\prime \prime} \in \mathbf{Y} \\ \mathbf{d}^{\prime}+\mathbf{d}^{\prime \prime}=\mathbf{d}}} \mathcal{X}\left(\mathbf{d}^{\prime}\right) \oplus \mathcal{Y}\left(\mathbf{d}^{\prime \prime}\right)
$$

is a presentation of $\bmod _{\Lambda}(\mathbf{d})$ as a finite disjoint sum of constructible sets of dimensions $a(\mathbf{d})+\left\langle\mathbf{d}^{\prime \prime}, \mathbf{d}^{\prime}\right\rangle$, respectively. Thus we have to show that the set of $M \in \mathcal{X}\left(\mathbf{d}^{\prime}\right) \oplus \mathcal{Y}\left(\mathbf{d}^{\prime \prime}\right)$ such that $[M, M]^{2} \neq 0$ has dimension at most $a(\mathbf{d})-2$ for all $\mathbf{d}^{\prime}$ and $\mathbf{d}^{\prime \prime}$ as above. Note that by Lemma $4.7 \operatorname{ext}^{1}\left(\mathcal{Y}\left(\mathbf{d}^{\prime \prime}\right), \mathcal{X}\left(\mathbf{d}^{\prime}\right)\right)=-\left\langle\mathbf{d}^{\prime \prime}, \mathbf{d}^{\prime}\right\rangle$. Since obviously $\operatorname{hom}\left(\mathcal{Y}\left(\mathbf{d}^{\prime \prime}\right), \mathcal{X}\left(\mathbf{d}^{\prime}\right)\right)=0$, we get $\operatorname{ext}^{2}\left(\mathcal{Y}\left(\mathbf{d}^{\prime \prime}\right), \mathcal{X}\left(\mathbf{d}^{\prime}\right)\right)=0$. This implies our claim if $\left\langle\mathbf{d}^{\prime \prime}, \mathbf{d}^{\prime}\right\rangle<0$.

Assume now that $\mathbf{d}^{\prime} \in \mathbf{X}, \mathbf{d}^{\prime \prime} \in \mathbf{Y}, \mathbf{d}^{\prime}+\mathbf{d}^{\prime \prime}=\mathbf{d}$ and $\left\langle\mathbf{d}^{\prime \prime}, \mathbf{d}^{\prime}\right\rangle=0$. If $\mathbf{d} \in \mathbf{X}$, then Proposition 4.5 implies that $\mathbf{d}^{\prime}=\mathbf{d}$ and $\mathbf{d}^{\prime \prime}=0$. Consequently, $[M, M]^{2}=0$ for all $M \in \mathcal{X}\left(\mathbf{d}^{\prime}\right) \oplus \mathcal{Y}\left(\mathbf{d}^{\prime \prime}\right)=\mathcal{X}(\mathbf{d})$. A similar argument applies if $\mathbf{d} \in \mathbf{Y}$. If $\bmod _{\Lambda}^{P}(\mathbf{d}) \neq \varnothing$ and $\bmod _{\Lambda}^{I}(\mathbf{d}) \neq \varnothing$, then we can use the previous lemma.

\section{INEQUALITIES}

Throughout this section, $\Lambda$ is a fixed canonical algebra of type $\mathbf{m}=\left(m_{1}, \ldots, m_{n}\right)$.

5.1. Our aim in this section is to prove the following inequalities.

Proposition. Let $\mathbf{d} \in \mathbf{R}$ and $\mathbf{d}^{\prime} \in \mathbf{P}$ be such that $\mathbf{d}^{\prime} \neq 0$ and $\mathbf{d}-\mathbf{d}^{\prime} \in \mathbf{R}+\mathbf{Q}$.

(1) If $\sum_{i \in[1, n]} \frac{1}{m_{i}-1}>2 n-5$, then $\left\langle\mathbf{d}-\mathbf{d}^{\prime}, \mathbf{d}^{\prime}\right\rangle<0$.

(2) If $\sum_{i \in[1, n]} \frac{1}{m_{i}-1}=2 n-5$, then $\left\langle\mathbf{d}-\mathbf{d}^{\prime}, \mathbf{d}^{\prime}\right\rangle \leq 0$. Moreover, if $p^{\mathbf{d}}>0$, then the above inequality is strict.

(3) If $\mathbf{m}=(2,2,2,2,2)$ and $\mathbf{d}$ is sincere, then $\left\langle\mathbf{d}-\mathbf{d}^{\prime}, \mathbf{d}^{\prime}\right\rangle<0$.

According to the results of the previous section, the above proposition implies the "positive" parts of Theorems 1.3, 1.4 and 1.5.

5.2. We start with a series of simple inequalities leading to our main result. The elementary proof of the first inequality is left to the reader.

Lemma. Let $d$ and $\delta_{1}, \ldots, \delta_{m}, m>0$, be nonnegative and such that $d=$ $\sum_{i \in[1, m]} \delta_{i}$. Then

$$
\sum_{i<j \in[1, m]} \delta_{i} \delta_{j} \leq \frac{m-1}{2 m} d^{2} .
$$

Moreover, equality holds if and only if $\delta_{i}=\frac{d}{m}$ for all $i \in[1, m]$. 
5.3. We will need the following variant of the above inequality.

Lemma. Let $d$ and $\delta_{1}, \ldots, \delta_{m}, m>2$, be nonnegative and such that $d=$ $\sum_{i \in[1, m]} \delta_{i}$. Then

$$
\sum_{i<j \in[1, m]} \delta_{i} \delta_{j} \leq \frac{1}{4}\left(d+d^{\prime}\right)^{2}-\frac{m-1}{2(m-2)} d^{2}
$$

where $d^{\prime}=\sum_{i \in[2, m-1]} \delta_{i}$. Moreover, equality holds if and only if $\delta_{1}=\delta_{m}=\frac{d-d^{\prime}}{2}$ and $\delta_{i}=\frac{d^{\prime}}{m-2}$ for all $i \in[2, m-1]$.

Proof. It follows by direct calculations that

$$
\sum_{i<j \in[1, m]} \delta_{i} \delta_{j}=\delta_{1} \delta_{m}+\left(d-d^{\prime}\right) d^{\prime}+\sum_{i<j \in[2, m-1]} \delta_{i} \delta_{j}
$$

Now the claim follows by applying the previous lemma to $\delta_{1} \delta_{m}$ and $\sum_{i<j \in[2, m-1]} \delta_{i} \delta_{j}$.

5.4. The next step is the following.

Lemma. Let $d, q$ and $\delta_{1}, \ldots, \delta_{m}, m \geq 2$, be nonnegative and such that $d=$ $\sum_{i \in[1, m]} \delta_{i}$ and $\delta_{1}, \delta_{m} \geq q$. Then

$$
\sum_{i<j \in[1, m]} \delta_{i} \delta_{j} \leq \begin{cases}\frac{m-1}{2 m} d^{2}, & m q \leq d \\ (d-q)^{2}-\frac{m-1}{2(m-2)}(d-2 q)^{2}, & m q>d .\end{cases}
$$

Moreover, in the first case equality holds if and only if $\delta_{i}=\frac{d}{m}$ for all $i \in[1, m]$, and in the second case equality holds if and only if $\delta_{1}=\delta_{m}=q$ and $\delta_{i}=\frac{d-2 q}{m-2}$ for all $i \in[2, m-1]$.

Note that $m q>d$ may hold only for $m>2$.

Proof. The claim for $m=2$ is an easy exercise, hence we may assume that $m>2$ and apply the previous lemma. Since $d^{\prime}=\sum_{i \in[2, m-1]} \delta_{i}$ varies from 0 to $d-2 q$, the maximal value of $\frac{1}{4} d^{2}+\frac{1}{2} d d^{\prime}-\frac{m}{4(m-2)} d^{\prime 2}$ is obtained for $d^{\prime}=\min \left(\frac{m-2}{m} d, d-2 q\right)$. This immediately implies our claim.

5.5. The following inequality is what we really need.

Lemma. Let $d, q$ and $\delta_{1}, \ldots, \delta_{m}, m \geq 2$, be nonnegative and such that $d=$ $\sum_{i \in[1, m]} \delta_{i}$ and $\delta_{1} \geq q$. Then

$$
-\delta_{m} q+\sum_{i<j \in[1, m]} \delta_{i} \delta_{j} \leq \begin{cases}-d q+\frac{m-1}{2 m}(d+q)^{2}, & (m-1) q \leq d, \\ -d q+d^{2}-\frac{m-1}{2(m-2)}(d-q)^{2}, & (m-1) q>d\end{cases}
$$

Moreover, in the first case equality holds if and only if $\delta_{i}=\frac{d+q}{m}$ for all $i \in[1, m-1]$ and $\delta_{m}=\frac{d-(m-1) q}{m}$, and in the second case equality holds if and only if $\delta_{1}=q$, $\delta_{m}=0$ and $\delta_{i}=\frac{d-q}{m-2}$ for all $i \in[2, m-1]$.

Proof. Let $\delta_{i}^{\prime}=\delta_{i}$ for $i \in[1, m-1]$ and $\delta_{m}^{\prime}=\delta_{m}+q$. Then

$$
-\delta_{m} q+\sum_{i<j \in[1, m]} \delta_{i} \delta_{j}=-d q+\sum_{i<j \in[1, m]} \delta_{i}^{\prime} \delta_{j}^{\prime},
$$

and we may apply the previous lemma. 
5.6. We will also need the following consequence of the previous inequality.

Corollary. Let $d, q$ and $\delta_{1}, \ldots, \delta_{m}, m \geq 2$, be nonnegative and such that $d=$ $\sum_{i \in[1, m]} \delta_{i}$ and $\delta_{1} \geq q$. Then

$$
-\delta_{m} q+\sum_{i<j \in[1, m]} \delta_{i} \delta_{j} \leq \frac{1}{2} d^{2}-\frac{1}{2} q^{2} .
$$

Moreover, if equality holds, then $q=d$.

Proof. If $q=0$, then the claim is obvious from the previous lemma; thus we assume that $q>0$. We first consider the case $(m-1) q \leq d$. In this case $\frac{1}{m} \geq \frac{q}{d+q}$. Using the previous lemma once more one easily gets that

$$
\left(\frac{1}{2} d^{2}-\frac{1}{2} q^{2}\right)-\left(-\delta_{m} q+\sum_{i<j \in[1, m]} \delta_{i} \delta_{j}\right) \geq \frac{1}{2 m}(d+q)^{2}-q^{2} \geq \frac{1}{2} q(d-q),
$$

hence the claim follows. On the other hand, if $(m-1) q>d$, then

$$
\left(\frac{1}{2} d^{2}-\frac{1}{2} q^{2}\right)-\left(-\delta_{m} q+\sum_{i<j \in[1, m]} \delta_{i} \delta_{j}\right) \geq \frac{1}{2(m-2)}(d-q)^{2},
$$

which finishes the proof.

5.7. For a fixed positive $d$ and integers $m_{1}, m_{2}, m_{3} \geq 2$, let $f$ be the function defined on the set of all 4 -tuples $\left(p, p_{1}, p_{2}, p_{3}\right)$ of nonnegative real numbers such that $p+p_{1}+p_{2}+p_{3}=d$ by $f\left(p, p_{1}, p_{2}, p_{3}\right)=g_{m_{1}}\left(p_{1}\right)+g_{m_{2}}\left(p_{2}\right)+g_{m_{3}}\left(p_{3}\right)$, where

$$
g_{m}(q)= \begin{cases}\frac{m-1}{2 m}(d+q)^{2}, & (m-1) q \leq d, \\ d^{2}-\frac{m-1}{2(m-2)}(d-q)^{2}, & (m-1) q>d .\end{cases}
$$

Our next aim is to prove the following.

Lemma. If $\frac{1}{m_{1}-1}+\frac{1}{m_{2}-1}+\frac{1}{m_{3}-1} \geq 1$, then $f\left(p, p_{1}, p_{2}, p_{3}\right) \leq 2 d^{2}$. Moreover, equality holds if and only if $\frac{1}{m_{1}-1}+\frac{1}{m_{2}-1}+\frac{1}{m_{3}-1}=1, p=0$ and there exists $i \in[1,3]$ and $\lambda$ such that $\frac{m_{i}-1}{m_{i}} d \leq \lambda \leq d, p_{i}=\frac{m_{i}}{m_{i}-1} \lambda-d$ and $p_{j}=d-\frac{m_{j}-2}{m_{j}-1} \lambda$ for $j \neq i$.

Proof. If we substitute $p=\xi^{2}$ and $p_{i}=\xi_{i}^{2}$ for $i \in[1,3]$, then we may replace $f$ by a function $F$ defined on the set of all 4 -tuples $\left(\xi, \xi_{1}, \xi_{2}, \xi_{3}\right)$ of real numbers such that $\xi^{2}+\xi_{1}^{2}+\xi_{2}^{2}+\xi_{3}^{2}=d$ by $F\left(\xi, \xi_{1}, \xi_{2}, \xi_{3}\right)=G_{m_{1}}\left(\xi_{1}\right)+G_{m_{2}}\left(\xi_{2}\right)+G_{m_{3}}\left(\xi_{3}\right)$, where

$$
G_{m}(\mu)= \begin{cases}\frac{m-1}{2 m}\left(d+\mu^{2}\right)^{2}, & (m-1) \mu^{2} \leq d, \\ d^{2}-\frac{m-1}{2(m-2)}\left(d-\mu^{2}\right)^{2}, & (m-1) \mu^{2}>d .\end{cases}
$$

By direct calculations one checks that $F$ is differentiable. Since the set considered is compact, $F$ possesses a maximum. Using Lagrange's multipliers method we know that, if $F$ has a maximum at $\left(\xi, \xi_{1}, \xi_{2}, \xi_{3}\right)$, then there exists $\lambda$ such that $\lambda \xi=0$ and $\xi_{i} H_{m_{i}}\left(\xi_{i}\right)=0$ for all $i \in[1,3]$, where

$$
H_{m}(\mu)= \begin{cases}\frac{m-1}{m}\left(d+\mu^{2}\right)-\lambda, & (m-1) \mu^{2} \leq d, \\ \frac{m-1}{m-2}\left(d-\mu^{2}\right)-\lambda, & (m-1) \mu^{2}>d .\end{cases}
$$


If $\xi \neq 0$, then $\lambda=0$ and it follows that either $\xi_{i}=0$ or $\xi_{i}^{2}=d$ for each $i$. Let $I$ be the set of all $i$ such that $\xi_{i}^{2}=d$. Then $d|I|+\xi=d$, hence $I=\varnothing$ and $\xi^{2}=d$. We have

$$
f(d, 0,0,0)=\left(\frac{3}{2}-\frac{1}{2 m_{1}}-\frac{1}{2 m_{2}}-\frac{1}{2 m_{3}}\right) d^{2}<2 d^{2},
$$

thus we may assume that $\xi=0$.

Let $I_{0}=\left\{i \mid \xi_{i}=0\right\}, I_{1}=\left\{i \mid 0<\xi^{2} \leq \frac{1}{m_{i}-1} d\right\}$ and $I_{2}=\left\{i \mid \frac{1}{m_{i}-1} d<\xi^{2} \leq d\right\}$. Up to symmetry we have to consider the following cases:

- $I_{0}=\{1,2\}$;

- $I_{0}=\{1\}, I_{1}=\{2,3\}$;

- $I_{0}=\{1\}, I_{1}=\{2\}, I_{2}=\{3\}$

- $I_{0}=\{1\}, I_{2}=\{2,3\}$;

- $I_{1}=\{1,2,3\}$;

- $I_{1}=\{1,2\}, I_{2}=\{3\}$

- $I_{1}=\{1\}, I_{2}=\{2,3\}$.

Note that our assumption $\xi=0$ implies that $I_{0} \neq\{1,2,3\}$. On the other hand $\frac{1}{m_{1}-1}+\frac{1}{m_{2}-1}+\frac{1}{m_{3}-1} \geq 1$ implies that $I_{2} \neq\{1,2,3\}$. For future use, let $\delta=$ $\frac{1}{m_{1}-1}+\frac{1}{m_{2}-1}+\frac{1}{m_{3}-1}$ and $\gamma=\frac{1}{m_{2}-1}+\frac{1}{m_{3}-1}$.

We start with the case $I_{0}=\{1,2\}$, thus $p_{1}=p_{2}=0$. Then obviously $p_{3}=d$ and

$$
f\left(p, p_{1}, p_{2}, p_{3}\right)=\left(2-\frac{1}{2 m_{1}}-\frac{1}{2 m_{2}}\right) d^{2}<2 d^{2} .
$$

Assume now that $I_{0}=\{1\}$ and $I_{1}=\{2,3\}$, thus $p_{1}=0$. Moreover, there exists $\lambda$ such that $p_{2}=\frac{m_{2}}{m_{2}-1} \lambda-d$ and $p_{3}=\frac{m_{3}}{m_{3}-1} \lambda-d$. Since $p+p_{1}+p_{2}+p_{3}=d$, it follows that $\lambda=\frac{3}{2+\gamma} d$. The inequality $p_{3} \leq \frac{1}{m_{3}-1} d$ implies that $\lambda \leq d$, hence $\gamma \geq 1$. By direct calculations we get

$$
f\left(p, p_{1}, p_{2}, p_{3}\right)=\left(2-\frac{1}{2 m_{1}}-\frac{3(\gamma-1)}{2(2+\gamma)}\right) d^{2}<2 d^{2} .
$$

Now let $I_{0}=\{1\}, I_{1}=\{2\}$ and $I_{3}=\{3\}$. Then $p_{1}=0$ and there exists $\lambda$ such that $p_{2}=\frac{m_{2}}{m_{2}-1} \lambda-d$ and $p_{3}=d-\frac{m_{3}-2}{m_{3}-1} \lambda$. It follows that $\lambda=\frac{1}{\gamma} d$. The inequality $p_{3}>\frac{1}{m_{3}-1} d$ implies that $\lambda<d$, hence $\gamma>1$. One calculates that

$$
f\left(p, p_{1}, p_{2}, p_{3}\right)=\left(2-\frac{1}{2 m_{1}}-\frac{\gamma-1}{2 \gamma}\right) d^{2}<2 d^{2} .
$$

We now consider the case $I_{0}=\{1\}$ and $I_{2}=\{2,3\}$. Then $p_{1}=0$, and there exists $\lambda$ such that $p_{2}=d-\frac{m_{2}-2}{m_{2}-1} \lambda$ and $p_{3}=d-\frac{m_{3}-2}{m_{3}-1} \lambda$. It follows that $\lambda=\frac{1}{2-\gamma} d$. We get

$$
f\left(p, p_{1}, p_{2}, p_{3}\right)=\left(2-\frac{\delta-1}{2(\delta-\gamma+1)(2-\gamma)}\right) d^{2} \leq 2 d^{2} .
$$

Note that $\gamma<2$ since in this case $m_{2}, m_{3}>2$. The inequality is strict if and only if $\delta>1$. Note that if $\delta=1$, then $\lambda=\frac{m_{1}-1}{m_{1}} d$ and $p_{1}=\frac{m_{1}}{m_{1}-1} \lambda-d$.

The next case is $I_{1}=\{1,2,3\}$. Then $p_{1}=\frac{m_{1}}{m_{1}-1} \lambda-d, p_{2}=\frac{m_{2}}{m_{2}-1} \lambda-d$ and $p_{3}=\frac{m_{3}}{m_{3}-1} \lambda-d$ for some $\lambda$. It follows that $\lambda=\frac{4}{3+\delta} d$. We get

$$
f\left(p, p_{1}, p_{2}, p_{3}\right)=\left(2-\frac{2(\delta-1)}{3+\delta}\right) d^{2} \leq 2 d^{2} .
$$

Equality holds if and only if $\delta=1$. If this is the case, then $\lambda=d, p_{2}=d-\frac{m_{2}-2}{m_{2}-1} \lambda$ and $p_{3}=d-\frac{m_{3}-2}{m_{3}-1} \lambda$. 
Assume now that $I_{1}=\{1,2\}$ and $I_{2}=\{3\}$. There exists $\lambda$ such that $p_{1}=$ $\frac{m_{1}}{m_{1}-1} \lambda-d, p_{2}=\frac{m_{2}}{m_{2}-1} \lambda-d$ and $p_{3}=d-\frac{m_{3}-2}{m_{3}-1} \lambda$. It follows that $\lambda=\frac{2}{1+\delta} d$. The inequality $p_{3}>\frac{1}{m_{3}-1} d$ implies that $\lambda<d$, hence $\delta>1$. We get

$$
f\left(p, p_{1}, p_{2}, p_{3}\right)=\left(2-\frac{\delta-1}{1+\delta}\right) d^{2}<2 d^{2} .
$$

Finally, let $I_{1}=\{1\}$ and $I_{2}=\{2,3\}$. There exists $\lambda$ such that $p_{1}=\frac{m_{1}}{m_{1}-1} \lambda-d$, $p_{2}=d-\frac{m_{2}-2}{m_{2}-1} \lambda$ and $p_{3}=d-\frac{m_{3}-2}{m_{3}-1} \lambda$. It follows that $(\delta-1) \lambda=0$. If $\delta>1$, then $\lambda=0$ and $p_{1}=-d<0$, which is impossible. Assume now that $\delta=1$. The inequalities $p_{1}>0$ and $p_{3}>\frac{1}{m_{3}-1} d$ imply that $\frac{m_{1}-1}{m_{1}} d<\lambda<d$. One also checks that in this case

$$
f\left(p, p_{1}, p_{2}, p_{3}\right)=2 d^{2}
$$

which finishes the proof.

5.8. Our first aim is to prove Proposition 5.1 in the following situation. Let $\mathbf{d} \in \mathbf{R}$ and $\mathbf{d}^{\prime} \in \mathbf{P}$ be such that $\mathbf{d}^{\prime} \neq 0, d_{\alpha}^{\prime}=d_{\alpha}, d_{\omega}^{\prime}=0, \mathbf{d}-\mathbf{d}^{\prime} \in \mathbb{N}^{\Delta_{0}}$ and $p_{i, j}^{\mathbf{d}}=0$ for all $i \in[1, n]$ and $j \in\left[1, m_{i}-1\right]$. For simplicity we write in this case $d, p$ and $p_{1}, \ldots$, $p_{n}$ instead of $d_{\alpha}, p^{\mathbf{d}}$ and $p_{1, m_{1}}^{\mathbf{d}}, \ldots, p_{n, m_{n}}^{\mathbf{d}}$, respectively. Note that

$$
\left\langle\mathbf{d}-\mathbf{d}^{\prime}, \mathbf{d}^{\prime}\right\rangle=-d^{2}-d p+\sum_{i \in[1, n]}\left(-d_{i, m_{i}-1}^{\prime} p_{i}+\sum_{j \in\left[1, m_{i}-1\right]}\left(d_{i, j-1}^{\prime}-d_{i, j}^{\prime}\right) d_{i, j}^{\prime}\right)
$$

and $d=p+\sum_{i \in[1, n]} p_{i}$. Let $\delta_{i, j}=d_{i, j-1}^{\prime}-d_{i, j}^{\prime}$ for $i \in[1, n]$ and $j \in\left[1, m_{i}\right]$. Then

$$
\left\langle\mathbf{d}-\mathbf{d}^{\prime}, \mathbf{d}^{\prime}\right\rangle=-d^{2}-d p+\sum_{i \in[1, n]} S_{i},
$$

where $S_{i}=-\delta_{i, m_{i}} p_{i}+\sum_{j<l \in\left[1, m_{i}\right]} \delta_{i, j} \delta_{i, l}$. Note that $\delta_{i, j} \geq 0$ for $i \in[1, n]$ and $j \in\left[1, m_{i}\right], \sum_{j \in[1, n]} \delta_{i, j}=d$ for $i \in[1, n]$, and $\delta_{i, 1}=d_{\alpha}^{\prime}-d_{i, 1}^{\prime} \geq d_{\alpha}-d_{i, 1}=p_{i}$ for $i \in[1, n]$.

Let $\mathfrak{O}$ be the set of all pairs $\left(\mathbf{d}, \mathbf{d}^{\prime}\right)$ such that $\mathbf{d} \in \mathbf{R}, p^{\mathbf{d}}=0, p_{i, j}^{\mathbf{d}}=0$ for $i \in[1, n], j \in\left[1, m_{i}-1\right], \mathbf{d}^{\prime} \in \mathbf{P}, d_{i, j-1}^{\prime}>d_{i, j}^{\prime}$ for $i \in[1, n]$ and $j \in\left[1, m_{i}-1\right]$, $d_{\omega}^{\prime}=0$, and $\mathbf{d}-\mathbf{d}^{\prime} \in \mathbb{N}^{\Delta_{0}}$.

5.9. Assume first that $n=3$. It follows from the above paragraph and Lemma 5.5 that

$$
\left\langle\mathbf{d}-\mathbf{d}^{\prime}, \mathbf{d}^{\prime}\right\rangle \leq-2 d^{2}+f\left(p, p_{1}, p_{2}, p_{3}\right),
$$

where $f$ is as in Subsection 5.7. Lemma 5.7 shows that if $\frac{1}{m_{1}-1}+\frac{1}{m_{2}-1}+\frac{1}{m_{3}-1} \geq 1$, then

$$
\left\langle\mathbf{d}-\mathbf{d}^{\prime}, \mathbf{d}^{\prime}\right\rangle \leq 0
$$

and if equality holds, then $\frac{1}{m_{1}-1}+\frac{1}{m_{2}-1}+\frac{1}{m_{3}-1}=1$ and $p^{\mathbf{d}}=0$, which finishes the proof of Proposition 5.1 in this case. Note also that if equality holds, then according to Lemmas 5.5 and $5.7,\left(\mathbf{d}, \mathbf{d}^{\prime}\right) \in \mathfrak{O}$.

5.10. As the next case, consider $\mathbf{m}=(2,2,2, m)$. It follows from Lemma 5.5 that

$$
S_{i} \leq \frac{1}{4} d^{2}-\frac{1}{2} d p_{i}+\frac{1}{4} p_{i}^{2}
$$

for $i \in[1,3]$, where we use notation introduced in Subsection 5.8. If $p_{4}=d$, then $p=p_{1}=p_{2}=p_{3}=0$, and it follows from Corollary 5.6 that $S_{4} \leq 0$, hence

$$
\left\langle\mathbf{d}-\mathbf{d}^{\prime}, \mathbf{d}^{\prime}\right\rangle \leq-\frac{1}{4} d^{2}<0 .
$$


On the other hand, if $d>p_{4}$, then again using Corollary 5.6,

$$
S_{4}<\frac{1}{2} d^{2}-\frac{1}{2} p_{4}^{2},
$$

hence

$$
\begin{aligned}
& \left\langle\mathbf{d}-\mathbf{d}^{\prime}, \mathbf{d}^{\prime}\right\rangle \\
& \quad<-\frac{3}{4} p^{2}-\frac{1}{4} p_{4}^{2}-p p_{1}-p p_{2}-p p_{3}-\frac{1}{2} p p_{4}-\frac{1}{2} p_{1} p_{2}-\frac{1}{2} p_{1} p_{3}-\frac{1}{2} p_{2} p_{3} \leq 0 .
\end{aligned}
$$

5.11. Assume now that $\mathbf{m}=(2,2,3,3)$. It follows from Lemma 5.5 that

$$
S_{i} \leq \frac{1}{4} d^{2}-\frac{1}{2} d p_{i}+\frac{1}{4} p_{i}^{2}
$$

for $i \in[1,2]$. If $2 p_{3} \leq d$ and $2 p_{4} \leq d$, then again using Lemma 5.5, we get

$$
S_{i} \leq \frac{1}{3} d^{2}-\frac{1}{3} d p_{i}+\frac{1}{3} p_{i}^{2}
$$

for $i \in[3,4]$, hence

$$
\begin{aligned}
& \left\langle\mathbf{d}-\mathbf{d}^{\prime}, \mathbf{d}^{\prime}\right\rangle \leq-\frac{5}{6} p^{2}-\frac{1}{12} p_{1}^{2}-\frac{1}{12} p_{2}^{2}-\frac{7}{6} p p_{1}-\frac{7}{6} p p_{2}-\frac{5}{6} p p_{3}-\frac{5}{6} p p_{4} \\
& -\frac{2}{3} p_{1} p_{2}-\frac{1}{3} p_{1} p_{3}-\frac{1}{3} p_{1} p_{4}-\frac{1}{3} p_{2} p_{3}-\frac{1}{3} p_{2} p_{4}-\frac{1}{6} p_{3}\left(d-2 p_{3}\right)-\frac{1}{6} p_{4}\left(d-2 p_{4}\right) \leq 0 .
\end{aligned}
$$

Moreover, if equality holds, then $p=p_{1}=p_{2}=0$ and $p_{3}=p_{4}$. In addition applying Lemma 5.5 once more we get that if equality holds, then $\left(\mathbf{d}, \mathbf{d}^{\prime}\right) \in \mathfrak{O}$.

As the next case, consider $2 p_{4}>d$, i.e., $p_{4}>p+p_{1}+p_{2}+p_{3}$. Then in particular $2 p_{3} \leq d$, hence

and

$$
S_{3} \leq \frac{1}{3} d^{2}-\frac{1}{3} d p_{3}+\frac{1}{3} p_{3}^{2}, \quad S_{4} \leq d p_{4}-p_{4}^{2},
$$

$$
\begin{aligned}
\left\langle\mathbf{d}-\mathbf{d}^{\prime}, \mathbf{d}^{\prime}\right\rangle & \leq-\frac{7}{6} p^{2}-\frac{5}{12} p_{1}^{2}-\frac{5}{12} p_{2}^{2}-\frac{1}{6} p_{3}^{2}-\frac{1}{6} p_{4}^{2}-\frac{11}{6} p p_{1}-\frac{11}{6} p p_{2} \\
& -\frac{5}{3} p p_{3}-\frac{1}{3} p p_{4}-\frac{4}{3} p_{1} p_{2}-\frac{7}{6} p_{1} p_{3}+\frac{1}{6} p_{1} p_{4}-\frac{7}{6} p_{2} p_{3}+\frac{1}{6} p_{2} p_{4}+\frac{1}{3} p_{3} p_{4} .
\end{aligned}
$$

One easily checks that the above expression is decreasing when considered as a function of $p_{4}$ for $p_{4}>p+p_{1}+p_{2}+p_{3}$. Moreover, for $p_{4}=p+p_{1}+p_{2}+p_{3}$ we get

$$
-\frac{5}{3} p^{2}-\frac{5}{12} p_{1}^{2}-\frac{5}{12} p_{2}^{2}-\frac{7}{3} p p_{1}-\frac{7}{3} p p_{2}-2 p p_{3}-\frac{4}{3} p_{1} p_{2}-p_{1} p_{3}-p_{2} p_{3},
$$

hence $\left\langle\mathbf{d}-\mathbf{d}^{\prime}, \mathbf{d}\right\rangle<0$ in this case.

5.12. The final case we have to consider is $\mathbf{m}=(2,2,2,2,2)$. Let $\mathfrak{O}^{\prime}$ be the set of all pairs $\left(\mathbf{d}, \mathbf{d}^{\prime}\right) \in \mathfrak{O}$ such that $\mathbf{d}=d \mathbf{e}_{i, 2}$ for a positive integer $d$ and some $i \in[1,5]$, $d_{\alpha}^{\prime} \in[1, d], d_{i, 1}^{\prime}=0, d_{j, 1}^{\prime}=\frac{1}{2} d_{\alpha}^{\prime}$ for $j \in[1,5], j \neq i$, and $d_{\omega}^{\prime}=0$.

Using Lemma 5.5 we get that

$$
S_{i} \leq \frac{1}{4} d^{2}-\frac{1}{2} d p_{i}+\frac{1}{4} p_{i}^{2}
$$

for $i \in[1,5]$, hence

$$
\left\langle\mathbf{d}-\mathbf{d}^{\prime}, \mathbf{d}^{\prime}\right\rangle=-\frac{3}{4} p^{2}-\sum_{i \in[1,5]} p p_{i}-\frac{1}{2} \sum_{i<j \in[1,5]} p_{i} p_{j} \leq 0 .
$$

Moreover, if equality holds, then $p=0$ and there exists $i \in[1,5]$ such that $p_{i}=d$ and $p_{j}=0$ for $j \in[1,5], j \neq i$. Finally, it follows from Lemma 5.5 that in the case of equality $\left(\mathbf{d}, \mathbf{d}^{\prime}\right) \in \mathfrak{O}^{\prime}$. 
5.13. We now show that we can reduce the proof of Proposition 5.1 to the special situation considered in the previous paragraphs. We first show that we may assume that $d_{\omega}^{\prime}=0$.

Lemma. Let $\mathbf{d} \in \mathbf{R}$ and $\mathbf{d}^{\prime} \in \mathbf{P}$ be such that $\mathbf{d}^{\prime} \neq 0$ and $\mathbf{d}-\mathbf{d}^{\prime} \in \mathbf{R}+\mathbf{Q}$. If $d_{\omega}^{\prime}>0$, then $\mathbf{d}^{\prime}-\mathbf{h} \in \mathbf{P}, \mathbf{d}^{\prime}-\mathbf{h} \neq 0, \mathbf{d}-\left(\mathbf{d}^{\prime}-\mathbf{h}\right) \in \mathbf{R}+\mathbf{Q}$, and

$$
\left\langle\mathbf{d}-\mathbf{d}^{\prime}, \mathbf{d}^{\prime}\right\rangle=\left\langle\mathbf{d}-\left(\mathbf{d}^{\prime}-\mathbf{h}\right), \mathbf{d}^{\prime}-\mathbf{h}\right\rangle \text {. }
$$

Proof. The former three assertions are obvious $\left(\mathbf{d}^{\prime}-\mathbf{h} \neq 0\right.$, since $\left.\mathbf{h} \notin \mathbf{P}\right)$, the latter follows by direct calculations.

5.14. The second reduction is the following.

Lemma. Let $\mathbf{d} \in \mathbf{R}$ and $\mathbf{d}^{\prime} \in \mathbf{P}$ be such that $\mathbf{d}^{\prime} \neq 0, \mathbf{d}-\mathbf{d}^{\prime} \in \mathbf{R}+\mathbf{Q}$ and $d_{\omega}^{\prime}=0$. If $i \in[1, n]$ and $j \in\left[1, m_{i}-1\right]$ are such that $p_{i, j}^{\mathbf{d}}>0$ and $p_{i, j}^{\mathbf{d}-\mathbf{d}^{\prime}}>0$, then $\mathbf{d}-\mathbf{e}_{i, j} \in \mathbf{R}$, $\left(\mathbf{d}-\mathbf{e}_{i, j}\right)-\mathbf{d}^{\prime} \in \mathbf{R}+\mathbf{Q}$ and

$$
\left\langle\mathbf{d}-\mathbf{d}^{\prime}, \mathbf{d}^{\prime}\right\rangle \leq\left\langle\left(\mathbf{d}-\mathbf{e}_{i, j}\right)-\mathbf{d}^{\prime}, \mathbf{d}^{\prime}\right\rangle .
$$

Moreover, if $\left(\mathbf{d}-\mathbf{e}_{i, j}, \mathbf{d}^{\prime}\right) \in \mathfrak{O}$, then the above inequality is strict.

Proof. Obviously, $p_{i, j}^{\mathbf{d}}>0$ implies that $\mathbf{d}-\mathbf{e}_{i, j} \in \mathbf{R}$. Similarly, $p_{i, j}^{\mathbf{d}-\mathbf{d}^{\prime}}>0$ implies that $\left(\mathbf{d}-\mathbf{e}_{i, j}\right)-\mathbf{d}^{\prime}=\left(\mathbf{d}-\mathbf{d}^{\prime}\right)-\mathbf{e}_{i, j} \in \mathbf{R}+\mathbf{Q}$. Moreover,

$$
\left\langle\left(\mathbf{d}-\mathbf{e}_{i, j}\right)-\mathbf{d}^{\prime}, \mathbf{d}^{\prime}\right\rangle-\left\langle\mathbf{d}-\mathbf{d}^{\prime}, \mathbf{d}^{\prime}\right\rangle=d_{i, j-1}^{\prime}-d_{i, j}^{\prime} \geq 0 .
$$

Finally, if $\left(\mathbf{d}-\mathbf{e}_{i, j}, \mathbf{d}^{\prime}\right) \in \mathfrak{O}$, then $d_{i, j-1}^{\prime}>d_{i, j}^{\prime}$, hence the above inequality is strict.

5.15. A more complicated version of the above reduction is the following.

Lemma. Let $\mathbf{d} \in \mathbf{R}$ and $\mathbf{d}^{\prime} \in \mathbf{P}$ be such that $\mathbf{d}^{\prime} \neq 0$, $\mathbf{d}-\mathbf{d}^{\prime} \in \mathbf{R}+\mathbf{Q}$ and $d_{\omega}^{\prime}=0$. If $i \in[1, n]$ and $j \in\left[1, m_{i}-1\right]$ are such that $p_{i, j}^{\mathbf{d}}>0, p_{i, l}^{\mathbf{d}}=0$ for all $l \in\left[j+1, m_{i}-1\right]$, and $p_{i, j}^{\mathbf{d}-\mathbf{d}^{\prime}}=0$, then $\mathbf{d}-\mathbf{e}_{i, j} \in \mathbf{R}, \mathbf{d}^{\prime}-\mathbf{e}_{i, j} \in \mathbf{P}, \mathbf{d}^{\prime}-\mathbf{e}_{i, j} \neq 0$, $\left(\mathbf{d}-\mathbf{e}_{i, j}\right)-\left(\mathbf{d}^{\prime}-\mathbf{e}_{i, j}\right) \in \mathbf{R}+\mathbf{Q}$ and

$$
\left\langle\mathbf{d}-\mathbf{d}^{\prime}, \mathbf{d}^{\prime}\right\rangle \leq\left\langle\left(\mathbf{d}-\mathbf{e}_{i, j}\right)-\left(\mathbf{d}^{\prime}-\mathbf{e}_{i, j}\right), \mathbf{d}^{\prime}-\mathbf{e}_{i, j}\right\rangle .
$$

Moreover, if $\left(\mathbf{d}-\mathbf{e}_{i, j}, \mathbf{d}^{\prime}-\mathbf{e}_{i, j}\right) \in \mathfrak{O}$, then the above inequality is strict.

Proof. Obviously, $\left(\mathbf{d}-\mathbf{e}_{i, j}\right)-\left(\mathbf{d}^{\prime}-\mathbf{e}_{i, j}\right)=\mathbf{d}-\mathbf{d}^{\prime} \in \mathbf{R}+\mathbf{Q}$ and $p_{i, j}^{\mathbf{d}}>0$ implies that $\mathbf{d}-\mathbf{e}_{i, j} \in \mathbf{R}$. Moreover, $\mathbf{d}^{\prime}-\mathbf{e}_{i, j} \neq 0$, since $\mathbf{e}_{i, j} \notin \mathbf{P}$. Note that

$$
d_{i, l}-d_{i, l}^{\prime}=p^{\mathbf{d}-\mathbf{d}^{\prime}}+\sum_{\substack{s \in[1, n] \\ s \neq i}} p_{s, m_{s}}^{\mathbf{d}-\mathbf{d}^{\prime}}+p_{i, l}^{\mathbf{d}-\mathbf{d}^{\prime}}
$$

for $l \in\left[1, m_{i}-1\right]$, and

$$
d_{i, m_{i}}-d_{i, m_{i}}^{\prime}=p^{\mathbf{d}-\mathbf{d}^{\prime}}+\sum_{s \in[1, n]} p_{s, m_{s}}^{\mathbf{d}-\mathbf{d}^{\prime}}+p_{\omega}^{\mathbf{d}-\mathbf{d}^{\prime}} .
$$

Thus our assumption implies that

$$
d_{i, j}-d_{i, j}^{\prime}=\min _{l \in\left[1, m_{i}\right]}\left(d_{i, l}-d_{i, l}^{\prime}\right) .
$$

In particular,

$$
d_{i, j}-d_{i, j}^{\prime} \leq d_{i, j+1}-d_{i, j+1}^{\prime},
$$


and the above inequality is strict if $j=m_{i}-1$ (note that $\mathbf{d}^{\prime} \neq 0$ implies that $\left.p_{\omega}^{\mathbf{d}-\mathbf{d}^{\prime}} \neq 0\right)$.

We now show that $\mathbf{d}^{\prime}-\mathbf{e}_{i, j} \in \mathbf{P}$. In order to do this we have to prove that $d_{i, j}^{\prime}-d_{i, j+1}^{\prime}>0$. If $j<m_{i}-1$, then using that $p_{i, j+1}^{\mathbf{d}}=0$ and (5.15.2) we get

$$
d_{i, j}^{\prime}-d_{i, j+1}^{\prime} \geq d_{i, j}-d_{i, j+1}=p_{i, j}^{\mathbf{d}}-p_{i, j+1}^{\mathbf{d}}=p_{i, j}^{\mathbf{d}}>0 .
$$

If $j=m_{i}-1$, then $d_{i, j+1}^{\prime}=d_{\omega}^{\prime}=0$, so we have to prove that $d_{i, j}^{\prime}>0$. Choose $l \in$ $\left[1, m_{i}\right]$ such that $p_{i, l}^{\mathbf{d}}=0$. It follows similarly as above that $d_{i, l}<d_{i, j}$. Using (5.15.1) we get

thus the claim follows.

$$
d_{i, j}^{\prime}>d_{i, l}^{\prime} \geq 0
$$

In order to prove the required inequality note that

$$
\begin{aligned}
\left\langle\left(\mathbf{d}-\mathbf{e}_{i, j}\right)-\left(\mathbf{d}^{\prime}-\mathbf{e}_{i, j}\right), \mathbf{d}^{\prime}-\mathbf{e}_{i, j}\right\rangle-\left\langle\mathbf{d}-\mathbf{d}^{\prime}, \mathbf{d}^{\prime}\right\rangle & \\
& =\left(d_{i, j+1}-d_{i, j+1}^{\prime}\right)-\left(d_{i, j}-d_{i, j}^{\prime}\right) ;
\end{aligned}
$$

hence the claim follows from (5.15.2). It also follows that if $j=m_{i}-1$, then the inequality is strict. Finally assume that $j \in\left[1, m_{i}-2\right]$ and $\left(\mathbf{d}-\mathbf{e}_{i, j}, \mathbf{d}^{\prime}-\mathbf{e}_{i, j}^{\prime}\right) \in \mathfrak{O}$. This implies that $d_{i, j}=d_{i, j+1}+1$ and $d_{i, j}^{\prime} \geq d_{i, j+1}^{\prime}+2$, which finishes the proof.

5.16. The last reduction is the following.

Lemma. Let $\mathbf{d} \in \mathbf{R}$ and $\mathbf{d}^{\prime} \in \mathbf{P}$ be such that $\mathbf{d}^{\prime} \neq 0, \mathbf{d}-\mathbf{d}^{\prime} \in \mathbf{R}+\mathbf{Q}, d_{\omega}^{\prime}=0$, and $p_{i, j}^{\mathbf{d}}=0$ for all $i \in[1, n]$ and $j \in\left[1, m_{i}-1\right]$. If $d_{\alpha}^{\prime}<d_{\alpha}$, then there exists $i \in[1, n]$ such that $\mathbf{d}-\mathbf{e}_{i, m_{i}} \in \mathbf{R},\left(\mathbf{d}-\mathbf{e}_{i, m_{i}}\right)-\mathbf{d}^{\prime} \in \mathbf{R}+\mathbf{Q}$ and

$$
\left\langle\mathbf{d}-\mathbf{d}^{\prime}, \mathbf{d}^{\prime}\right\rangle \leq\left\langle\left(\mathbf{d}-\mathbf{e}_{i, m_{i}}\right)-\mathbf{d}^{\prime}, \mathbf{d}^{\prime}\right\rangle \text {. }
$$

Moreover, if $\left(\mathbf{d}-\mathbf{e}_{i, m_{i}}, \mathbf{d}^{\prime}\right) \in \mathfrak{O}$, then $\left(\mathbf{d}, \mathbf{d}^{\prime}\right) \in \mathfrak{O}$. Finally, if $\mathbf{m}=(2,2,2,2,2)$, $\left(\mathbf{d}-\mathbf{e}_{i, m_{i}}, \mathbf{d}^{\prime}\right) \in \mathfrak{O}^{\prime}$ and $\left(\mathbf{d}, \mathbf{d}^{\prime}\right) \notin \mathfrak{O}^{\prime}$, then the above inequality is strict.

Proof. We first show the existence of $i \in[1, n]$ such that $\mathbf{d}-\mathbf{e}_{i, m_{i}} \in \mathbf{R}$ and

$$
\left(\mathbf{d}-\mathbf{e}_{i, m_{i}}\right)-\mathbf{d}^{\prime} \in \mathbf{R}+\mathbf{Q} .
$$

Observe that $0<d_{\alpha}^{\prime}<d_{\alpha}=p^{\mathbf{d}}+\sum_{i \in[1, n]} p_{i, m_{i}}^{\mathbf{d}}$, hence either $p^{\mathbf{d}}>0$ or there exists $i \in[1, n]$ such that $p_{i, m_{i}}^{\mathbf{d}}>0$. Similarly, since $d_{\alpha}-d_{\alpha}^{\prime}>0$, either $p^{\mathbf{d}-\mathbf{d}^{\prime}}>0$ or there exists $i \in[1, n]$ such that $p_{i, m_{i}}^{\mathbf{d}-\mathbf{d}^{\prime}}>0$. Note that if $p^{\mathbf{d}}>0$, then $\mathbf{d}-\mathbf{e}_{i, m_{i}} \in \mathbf{R}$ for all $i \in[1, n]$, since $\mathbf{h}-\mathbf{e}_{i, m_{i}}=\sum_{j \in\left[1, m_{i}-1\right]} \mathbf{e}_{i, j}$. Again similarly, if $p^{\mathbf{d}-\mathbf{d}^{\prime}}>0$, then $\left(\mathbf{d}-\mathbf{e}_{i, m_{i}}\right)-\mathbf{d}^{\prime}=\left(\mathbf{d}-\mathbf{d}^{\prime}\right)-\mathbf{e}_{i, m_{i}} \in \mathbf{R}+\mathbf{Q}$ for all $i \in[1, n]$. Thus it remains to show that if $p^{\mathbf{d}}=0=p^{\mathbf{d}-\mathbf{d}^{\prime}}$, then there exists $i \in[1, n]$ such that $p_{i, m_{i}}^{\mathbf{d}}, p_{i, m_{i}}^{\mathbf{d}-\mathbf{d}^{\prime}}>0$. Without loss of generality we may assume that $p_{1, m_{1}}^{\mathbf{d}}, \ldots, p_{s, m_{s}}^{\mathbf{d}}>$ 0 and $p_{s+1, m_{s+1}}^{\mathbf{d}}=\cdots=p_{n, m_{n}}^{\mathbf{d}}=0$ for some $s \in[1, n]$. Then for $i \in[s+1, n]$ and $j \in\left[1, m_{i}-1\right], d_{i, j}=d_{\alpha}$ and $\left(d_{i, j}-d_{i, j}^{\prime}\right)-\left(d_{\alpha}-d_{\alpha}^{\prime}\right)=p_{i, j}^{\mathbf{d}-\mathbf{d}^{\prime}}-p_{i, m_{i}}^{\mathbf{d}-\mathbf{d}^{\prime}}$, hence $p_{i, m_{i}}^{\mathbf{d}-\mathbf{d}^{\prime}}-p_{i, j}^{\mathbf{d}-\mathbf{d}^{\prime}}=d_{i, j}^{\prime}-d_{\alpha}^{\prime} \leq 0$. Consequently,

$$
p_{i, m_{i}}^{\mathbf{d}-\mathbf{d}^{\prime}}=\min \left\{p_{i, j}^{\mathbf{d}-\mathbf{d}^{\prime}} \mid j \in\left[1, m_{i}\right]\right\}=0
$$

for $i \in[s+1, n]$. Since $d_{\alpha}-d_{\alpha}^{\prime}>0$, it follows that there exists $i \in[1, s]$ such that $p_{i, m_{i}}^{\mathbf{d}-\mathbf{d}^{\prime}}>0$.

Note that

$$
\left\langle\left(\mathbf{d}-\mathbf{e}_{i, m_{i}}\right)-\mathbf{d}^{\prime}, \mathbf{d}^{\prime}\right\rangle-\left\langle\mathbf{d}-\mathbf{d}^{\prime}, \mathbf{d}^{\prime}\right\rangle=d_{i, m_{i}-1}^{\prime} \geq 0 .
$$


Obviously, if $\left(\mathbf{d}-\mathbf{e}_{i, m_{i}}, \mathbf{d}^{\prime}\right) \in \mathfrak{O}$, then $\left(\mathbf{d}, \mathbf{d}^{\prime}\right) \in \mathfrak{O}$. Finally, assume that $\mathbf{m}=$ $(2,2,2,2,2)$ and $\left(\mathbf{d}-\mathbf{e}_{i, m_{i}}, \mathbf{d}^{\prime}\right) \in \mathfrak{O}^{\prime}$. In particular, $\mathbf{d}-\mathbf{e}_{i, m_{i}}=d \mathbf{e}_{j, m_{j}}$ for a positive integer $d$ and $j \in[1, n]$. If $\left(\mathbf{d}, \mathbf{d}^{\prime}\right) \notin \mathfrak{O}^{\prime}$, then $j \neq i$, hence $d_{i, m_{i}-1}^{\prime}=\frac{1}{2} d \neq 0$, and the above inequality is strict, which finishes the proof.

5.17. We can now complete the proof of Proposition 5.1. Let $\Lambda$ be a canonical algebra of type $\mathbf{m}, \mathbf{d} \in \mathbf{R}$ and let $\mathbf{d}^{\prime} \in \mathbf{P}$ be such that $\mathbf{d}^{\prime} \neq 0$ and $\mathbf{d}-\mathbf{d}^{\prime} \in \mathbf{R}+\mathbf{Q}$. It follows from Lemma 5.13 that we may assume $d_{\omega}^{\prime}=0$. It follows by an easy induction that there exists a sequence $\left(\mathbf{d}^{(s)}, \mathbf{d}^{\prime(s)}\right), s \in[0, l]$, such that $\mathbf{d}^{(0)}=\mathbf{d}$, $\mathbf{d}^{\prime(0)}=\mathbf{d}^{\prime},\left(\mathbf{d}^{(s)}, \mathbf{d}^{\prime(s)}\right)$ is obtained from $\left(\mathbf{d}^{(s-1)}, \mathbf{d}^{\prime(l-s)}\right), s \in[1, l]$, by applying one of the reductions described in Lemmas 5.14-5.16, $d_{\alpha}^{(l)}=d_{\alpha}^{(l)}$, and $p_{i, j}^{\mathbf{d}^{(l)}}=0$ for all $i \in[1, n]$ and $j \in\left[1, m_{i}-1\right]$. In particular we know that

$$
\left\langle\mathbf{d}-\mathbf{d}^{\prime}, \mathbf{d}^{\prime}\right\rangle \leq\left\langle\mathbf{d}^{(l)}-\mathbf{d}^{(l)}, \mathbf{d}^{\prime(l)}\right\rangle \leq 0,
$$

where the latter inequality follows from Subsections 5.9-5.12. Moreover, the latter inequality is strict if $\sum_{i \in[1, n]} \frac{1}{m_{i}-1}>2 n-5$.

Now assume that $\sum_{i \in[1, n]} \frac{1}{m_{i}-1}=2 n-5$ and $\left\langle\mathbf{d}-\mathbf{d}^{\prime}, \mathbf{d}^{\prime}\right\rangle=0$. Then $\left\langle\mathbf{d}^{(l)}-\right.$ $\left.\mathbf{d}^{\prime(l)}, \mathbf{d}^{\prime(l)}\right\rangle=0$ and consequently

$$
\left\langle\mathbf{d}^{(s-1)}-\mathbf{d}^{\prime(s-1)}, \mathbf{d}^{\prime(s-1)}\right\rangle=\left\langle\mathbf{d}^{(s)}-\mathbf{d}^{\prime(s)}, \mathbf{d}^{\prime(s)}\right\rangle
$$

for all $s \in[1, l]$. It also follows from Subsections $5.9,5.11$ and 5.12 that $\left(\mathbf{d}^{(l)}, \mathbf{d}^{\prime(l)}\right) \in$ $\mathfrak{O}$, hence using Lemmas 5.14-5.16 we get by induction that for all $s \in[0, l]$, $\left(\mathbf{d}^{(s)}, \mathbf{d}^{\prime(s)}\right) \in \mathfrak{O}$. In particular, $p^{\mathbf{d}}=p^{\mathbf{d}^{(0)}}=0$. With similar arguments we prove that $\left(\mathbf{d}, \mathbf{d}^{\prime}\right) \in \mathfrak{O}^{\prime}$ if $\mathbf{m}=(2,2,2,2,2)$ and $\left\langle\mathbf{d}-\mathbf{d}^{\prime}, \mathbf{d}^{\prime}\right\rangle=0$, which implies the last assertion of Proposition 5.1.

\section{Counterexamples}

In this section we present for a canonical algebra of type $\mathbf{m}$ such that

$$
\sum_{i \in[1, n]} \frac{1}{m_{i}-1}=2 n-5(<2 n-5, \text { respectively }),
$$

examples of dimension vectors $\mathbf{d}^{\prime} \in \mathbf{P}$ and $\mathbf{d}^{\prime \prime} \in \mathbf{Q}$ such that $\mathbf{d}^{\prime}+\mathbf{d}^{\prime \prime} \in \mathbf{R}$ and

$$
\left\langle\mathbf{d}^{\prime \prime}, \mathbf{d}^{\prime}\right\rangle=0 \text { ( }>0 \text {, respectively). }
$$

Together with Propositions 4.3, 4.5, 4.9 and 5.1, it will finish the proof of Theorems $1.3,1.4$ and 1.5.

6.1. Let $\Lambda$ be a canonical algebra of type $\left(m_{1}, m_{2}, m_{3}\right)$ such that

$$
\delta=\frac{1}{m_{1}-1}+\frac{1}{m_{2}-1}+\frac{1}{m_{3}-1} \leq 1 .
$$

Note that our assumption implies that $m_{1}, m_{2}, m_{3}>2$. Let

$$
m=\left(m_{1}-1\right)\left(m_{2}-1\right)\left(m_{3}-1\right)\left(m_{1}-2\right)\left(m_{2}-2\right)\left(m_{3}-2\right) .
$$

Define $\mathbf{d}^{\prime}$ and $\mathbf{d}^{\prime \prime}$ by

$$
\begin{array}{rlrl}
d_{\alpha}^{\prime} & =\delta m, & d_{\alpha}^{\prime \prime} & =0, \\
d_{i, j}^{\prime}=\frac{\left(\delta\left(m_{i}-1\right)-1\right)\left(m_{i}-j-1\right)}{\left(m_{i}-1\right)\left(m_{i}-2\right)} m, & d_{i, j}^{\prime \prime}= & \frac{\left(\delta\left(m_{i}-1\right)-1\right)(j-1)}{\left(m_{i}-1\right)\left(m_{i}-2\right)} m, \\
& & i \in[1,3], j \in\left[1, m_{i}-1\right], \\
d_{\omega}^{\prime}=0, & d_{\omega}^{\prime \prime}=\delta m .
\end{array}
$$


Then $\mathbf{d}^{\prime} \in \mathbf{P}, \mathbf{d}^{\prime \prime} \in \mathbf{Q}$,

$$
\mathbf{d}^{\prime}+\mathbf{d}^{\prime \prime}=\frac{m}{m_{1}-1} \mathbf{e}_{1, m_{1}}+\frac{m}{m_{2}-1} \mathbf{e}_{2, m_{2}}+\frac{m}{m_{3}-1} \mathbf{e}_{3, m_{3}} \in \mathbf{R}
$$

and

$$
\begin{aligned}
\left\langle\mathbf{d}^{\prime \prime}, \mathbf{d}^{\prime}\right\rangle & =\sum_{i \in[1,3]} \sum_{j \in\left[2, m_{i}-1\right]} d_{i, j}^{\prime \prime}\left(d_{i, j}^{\prime}-d_{i, j-1}^{\prime}\right)+d_{\omega}^{\prime \prime} d_{\alpha}^{\prime} \\
& =\frac{1}{2}\left(-\sum_{i \in[1,3]} \frac{\left(\delta\left(m_{i}-1\right)-1\right)^{2}}{\left(m_{i}-1\right)\left(m_{i}-2\right)}+2 \delta^{2}\right) m^{2} \\
& =\frac{1}{2}\left(-\delta^{2}-\delta^{2} \delta^{\prime}+2 \delta \delta^{\prime}+\delta-\delta^{\prime}\right) m^{2}=\frac{1}{2}(1-\delta)\left(\delta \delta^{\prime}+\delta-\delta^{\prime}\right) \\
& =\frac{1}{2}(1-\delta)\left(\sum_{i \neq j \in[1,3]} \frac{1}{\left(m_{i}-1\right)\left(m_{j}-2\right)}\right) m^{2} \geq 0,
\end{aligned}
$$

where

$$
\delta^{\prime}=\frac{1}{m_{1}-2}+\frac{1}{m_{2}-2}+\frac{1}{m_{3}-2} .
$$

The above inequality is strict if $\delta<1$.

6.2. Let $\Lambda$ be a canonical algebra of type $\left(m_{1}, m_{2}, m_{3}, m_{4}\right)$ such that

$$
\frac{1}{m_{1}-1}+\frac{1}{m_{2}-1}+\frac{1}{m_{3}-1}+\frac{1}{m_{4}-1} \leq 3 \text {. }
$$

The above assumption implies in particular that, without loss of generality, we may assume that $m_{3}, m_{4}>2$. Let

$$
m=2 m_{1} m_{2}\left(m_{3}-2\right)\left(m_{4}-2\right) .
$$

Define $\mathbf{d}^{\prime}$ and $\mathbf{d}^{\prime \prime}$ by

$$
\begin{aligned}
& d_{\alpha}^{\prime}=m, \quad d_{\alpha}^{\prime \prime}=0, \\
& d_{i, j}^{\prime}=\frac{m_{i}-j}{m_{i}} m, \quad d_{i, j}^{\prime \prime}=\frac{j}{m_{i}} m, \quad i \in[1,2], j \in\left[1, m_{i}-1\right] \text {, } \\
& d_{i, j}^{\prime}=\frac{m_{i}-j-1}{2\left(m_{i}-2\right)} m, \quad d_{i, j}^{\prime \prime}=\frac{j-1}{2\left(m_{i}-2\right)} m, \quad i \in[3,4], j \in\left[1, m_{i}-1\right], \\
& d_{\omega}^{\prime}=0, \quad d_{\omega}^{\prime \prime}=m \text {. }
\end{aligned}
$$

Then $\mathbf{d}^{\prime} \in \mathbf{P}, \mathbf{d}^{\prime \prime} \in \mathbf{Q}$,

$$
\mathbf{d}^{\prime}+\mathbf{d}^{\prime \prime}=\frac{m}{2} \mathbf{e}_{3, m_{3}}+\frac{m}{2} \mathbf{e}_{3, m_{4}} \in \mathbf{R}
$$

and

$$
\left\langle\mathbf{d}^{\prime \prime}, \mathbf{d}^{\prime}\right\rangle=\left(\frac{3}{4}-\frac{1}{2 m_{1}}-\frac{1}{2 m_{2}}-\frac{1}{8\left(m_{3}-2\right)}-\frac{1}{8\left(m_{4}-2\right)}\right) m^{2} \geq 0 .
$$

The inequality is strict if $\mathbf{m} \neq(2,2,3,3)$.

6.3. Let $\Lambda$ be a canonical algebra of type $\left(m_{1}, \ldots, m_{n}\right)$ for $n \geq 5$. We may assume, without loss of generality, that $m_{n}=\min \left(m_{1}, \ldots, m_{n}\right)$. Let

$$
m=m_{1} \cdots m_{n-1} \text {. }
$$


Define $\mathbf{d}^{\prime}$ and $\mathbf{d}^{\prime \prime}$ by

$$
\begin{aligned}
& d_{\alpha}^{\prime}=m, \quad d_{\alpha}^{\prime \prime}=0, \\
& d_{i, j}^{\prime}=\frac{m_{i}-j}{m_{i}} m, \quad d_{i, j}^{\prime \prime}=\frac{j}{m_{i}} m, \quad i \in[1, n-1], j \in\left[1, m_{i}-1\right], \\
& d_{n, j}^{\prime}=0, \quad \quad d_{n, j}^{\prime \prime}=0, \quad j \in\left[1, m_{n}-1\right], \\
& d_{\omega}^{\prime}=0, \quad d_{\omega}^{\prime \prime}=m .
\end{aligned}
$$

Then $\mathbf{d}^{\prime} \in \mathbf{P}, \mathbf{d}^{\prime \prime} \in \mathbf{Q}$,

$$
\mathbf{d}^{\prime}+\mathbf{d}^{\prime \prime}=m \mathbf{e}_{n, m_{n}}
$$

and

$$
\left\langle\mathbf{d}^{\prime \prime}, \mathbf{d}^{\prime}\right\rangle=\frac{1}{2}\left(n-3-\sum_{i \in[1, n-1]} \frac{1}{m_{i}}\right) m^{2} \geq 0 .
$$

The inequality is strict if $\mathbf{m} \neq(2,2,2,2,2)$ (remember that $m_{n}=\min \left(m_{1}, \ldots, m_{n}\right)$ ).

6.4. Note that in all the above examples $p^{\mathbf{d}}=0$ for $\mathbf{d}=\mathbf{d}^{\prime}+\mathbf{d}^{\prime \prime}$. Moreover, $\mathbf{d}$ is not sincere for $n \geq 5$. In order to complete the proof of Theorem 1.5 we have to present examples with $p^{\mathbf{d}}>0$ and $\left\langle\mathbf{d}^{\prime \prime}, \mathbf{d}^{\prime}\right\rangle>0$, for canonical algebras $\Lambda$ of type $\left(m_{1}, \ldots, m_{n}\right)$ with $\sum_{i \in[1, n]} \frac{1}{m_{i}-1}<2 n-5$. It will also complete the proof of Theorem 1.4, since $\mathbf{d} \in \mathbf{R}$ with $p^{\mathbf{d}}>0$ is sincere.

Let $\Lambda$ be an algebra of the above form. It follows from the preceding subsections that there exist dimension vectors $\mathbf{d}^{\prime} \in \mathbf{P}$ and $\mathbf{d}^{\prime \prime} \in \mathbf{Q}$ such that $\mathbf{d}^{\prime}+\mathbf{d}^{\prime \prime} \in \mathbf{R}$ and $\left\langle\mathbf{d}^{\prime \prime}, \mathbf{d}^{\prime}\right\rangle>0$. Choose a positive integer $q$ such that

$$
q\left\langle\mathbf{d}^{\prime \prime}, \mathbf{d}^{\prime}\right\rangle+\left\langle\mathbf{d}^{\prime \prime}, \mathbf{h}\right\rangle>0 .
$$

Then $\hat{\mathbf{d}}^{\prime}=q \mathbf{d}^{\prime}+\mathbf{h} \in \mathbf{P}, \hat{\mathbf{d}}^{\prime \prime}=q \mathbf{d}^{\prime \prime} \in \mathbf{Q}, \mathbf{d}=\hat{\mathbf{d}}^{\prime}+\hat{\mathbf{d}}^{\prime \prime}=\mathbf{h}+q\left(\mathbf{d}^{\prime}+\mathbf{d}^{\prime \prime}\right) \in \mathbf{R}, p^{\mathbf{d}}>0$ and

$$
\left\langle\hat{\mathbf{d}}^{\prime \prime}, \hat{\mathbf{d}}^{\prime}\right\rangle=q^{2}\left\langle\mathbf{d}^{\prime \prime}, \mathbf{d}^{\prime}\right\rangle+q\left\langle\mathbf{d}^{\prime \prime}, \mathbf{h}\right\rangle>0
$$

\section{REFERENCES}

[1] M Barot and J. Schröer, Module varieties over canonical algebras, J. Algebra 246 (2001), no. 1, 175-192. MR1872616 (2003e:16013)

[2] G. Bobiński and A. Skowroński, Geometry of modules over tame quasi-tilted algebras, Colloq. Math. 79 (1999), no. 1, 85-118. MR1671811 (2000i:14067)

[3] Geometry of periodic modules over tame concealed and tubular algebras, Algebr. Represent. Theory 5 (2002), no. 2, 187-200. MR1909550 (2003d:16021)

[4] K. Bongartz, Algebras and quadratic forms, J. London Math. Soc. (2) 28 (1983), no. 3, 461469. MR724715 (85i:16036)

[5] - Minimal singularities for representations of Dynkin quivers, Comment. Math. Helv. 69 (1994), no. 4, 575-611. MR1303228 (96f:16016)

[6] On degenerations and extensions of finite-dimensional modules, Adv. Math. 121 (1996), no. 2, 245-287. MR1402728 (98e:16012)

[7] Some geometric aspects of representation theory, Algebras and Modules, I (Trondheim, 1996), 1998, pp. 1-27. MR1648601 (99j:16005)

[8] W. Crawley-Boevey and J. Schröer, Irreducible components of varieties of modules, J. Reine Angew. Math. 553 (2002), 201-220. MR1944812 (2004a:16020)

[9] M. Domokos and H. Lenzing, Invariant theory of canonical algebras, J. Algebra 228 (2000), no. 2, 738-762. MR1764590 (2001h:16016)

[10] Moduli spaces for representations of concealed-canonical algebras, J. Algebra 251 (2002), no. 1, 371-394. MR1900290 (2003d:16016) 
[11] W. Geigle and H. Lenzing, A class of weighted projective curves arising in representation theory of finite-dimensional algebras, Singularities, Representation of Algebras, and Vector Bundles (Lambrecht, 1985), 1987, pp. 265-297. MR915180 (89b:14049)

[12] Ch. Geiß, Geometric methods in representation theory of finite-dimensional algebras, Representation Theory of Algebras and Related Topics (Mexico City, 1994), 1996, pp. 53-63.

[13] D. Happel, A characterization of hereditary categories with tilting object, Invent. Math. 144 (2001), no. 2, 381-398. MR1827736 (2002a:18014)

[14] D. Happel, I. Reiten, and S. Smalø, Tilting in abelian categories and quasitilted algebras, Mem. Amer. Math. Soc. 120 (1996), no. 575, viii+ 88. MR1327209 (97j:16009)

[15] H. Kraft, Geometrische Methoden in der Invariantentheorie, Aspects of Mathematics, D1, Friedr. Vieweg \& Sohn, Braunschweig, 1984. MR768181 (86j:14006)

[16] - Geometric methods in representation theory, Representations of Algebras (Puebla, 1980), 1982, pp. 180-258. MR672117 (84c:14007)

[17] H. Lenzing and J. A. de la Peña, Concealed-canonical algebras and separating tubular families, Proc. London Math. Soc. (3) 78 (1999), no. 3, 513-540. MR1674837 (2000c:16018)

[18] M. Reineke, The monoid of families of quiver representations, Proc. London Math. Soc. (3) 84 (2002), no. 3, 663-685. MR1888427 (2004d:16030)

[19] C. M. Ringel, The rational invariants of the tame quivers, Invent. Math. 58 (1980), no. 3, 217-239. MR571574 (81f:16048)

[20] - Tame Algebras and Integral Quadratic Forms, Lecture Notes in Mathematics, vol. 1099, Springer-Verlag, Berlin, 1984. MR774589 (87f:16027)

[21] A. Skowroński, On omnipresent tubular families of modules, Representation Theory of Algebras (Cocoyoc, 1994), 1996, pp. 641-657. MR1388078 (97f:16032)

[22] A. Skowroński and J. Weyman, Semi-invariants of canonical algebras, Manuscripta Math. 100 (1999), no. 3, 391-403. MR1726226 (2001d:16026)

[23] D. Voigt, Induzierte Darstellungen in der Theorie der endlichen, algebraischen Gruppen, Lecture Notes in Mathematics, vol. 592, Springer-Verlag, Berlin, 1977. MR0486168 (58:5949)

Faculty of Mathematics and Computer Science, Nicolaus Copernicus University, Ul. Chopina 12/18, 87-100 Toruń, Poland

E-mail address: gregbob@mat.uni.torun.pl 